\title{
Antioxidant Potential of Adiponectin and Full PPAR- $\gamma$ Agonist in Correcting Streptozotocin-Induced Vascular Abnormality in Spontaneously Hypertensive Rats
}

\author{
Sheryar Afzal $\mathbb{D}^{1,2}$ Munavvar Abdul Sattar $\mathbb{D}^{1,2}$ Edward James Johns, ${ }^{2}$ \\ Olorunfemi A. Eseyin $\mathbb{C}^{2},{ }^{2}$ and Ali Attiq $\oplus^{1}$ \\ ${ }^{1}$ Department of Pharmacology \& Toxicology, Faculty of Pharmacy, MAHSA University, Selangor, Malaysia \\ ${ }^{2}$ School of Pharmaceutical Sciences, University Sains Malaysia, Penang, Malaysia \\ Correspondence should be addressed to Sheryar Afzal; sheryarafzal@mahsa.edu.my
}

Received 24 December 2020; Revised 15 July 2021; Accepted 12 August 2021; Published 14 October 2021

Academic Editor: Stéphane Mandard

Copyright (C) 2021 Sheryar Afzal et al. This is an open access article distributed under the Creative Commons Attribution License, which permits unrestricted use, distribution, and reproduction in any medium, provided the original work is properly cited.

\begin{abstract}
Oxidative stress, which is associated with metabolic and anthropometric perturbations, leads to reactive oxygen species production and decrease in plasma adiponectin concentration. We investigated pharmacodynamically the pathophysiological role and potential implication of exogenously administered adiponectin with full and partial peroxisome proliferatoractivated receptor-gamma (PPAR- $\gamma$ ) agonists on modulation of oxidative stress, metabolic dysregulation, and antioxidant potential in streptozotocin-induced spontaneously hypertensive rats (SHR). Group I (WKY) serves as the normotensive control, whereas 42 male SHRs were randomized equally into 7 groups $(n=6)$; group II serves as the SHR control, group III serves as the SHR diabetic control, and groups IV, V, and VI are treated with irbesartan $(30 \mathrm{mg} / \mathrm{kg})$, pioglitazone $(10 \mathrm{mg} / \mathrm{kg})$, and adiponectin $(2.5 \mu \mathrm{g} / \mathrm{kg})$, whereas groups VII and VIII received cotreatments as irbesartan+adiponectin and pioglitazone+adiponectin, respectively. Diabetes was induced using an intraperitoneal injection of streptozotocin ( $40 \mathrm{mg} / \mathrm{kg})$. Plasma adiponectin, lipid contents, and arterial stiffness with oxidative stress biomarkers were measured using an in vitro and in vivo analysis. Diabetic SHRs exhibited hyperglycemia, hypertriglyceridemia, hypercholesterolemia, and increased arterial stiffness with reduced plasma adiponectin and antioxidant enzymatic levels $(P<0.05)$. Diabetic SHRs pretreated with pioglitazone and adiponectin separately exerted improvements in antioxidant enzyme activities, abrogated arterial stiffness, and offset the increased production of reactive oxygen species and dyslipidemic effects of STZ, whereas the blood pressure values were significantly reduced in the irbesartan-treated groups (all $P<0.05$ ). The combined treatment of exogenously administered adiponectin with full PPAR- $\gamma$ agonist augmented the improvement in lipid contents and adiponectin concentration and restored arterial stiffness with antioxidant potential effects, indicating the degree of synergism between adiponectin and full PPAR- $\gamma$ agonists (pioglitazone).
\end{abstract}

\section{Introduction}

Diabetes mellitus (DM) and hypertension (HTN) are being considered foremost public health and medical issues; therefore, their coexistence has received greater attention because of rising epidemic globally as common chronic diseases, which associate mainly with micro- and macrocardiovascular complications [1], thus accelerating hyperglycemic issues in diabetic individuals [2]. Previous studies also signify the role of hyperglycemia leading to oxidative stress (OS) with endothelial dysfunction in blood vessels of type 1 diabetic patients [3]. Additionally, the concepts of oxidative stress and endothelial dysfunction have gained interest in recent years as contributing factors in the pathogenesis of hypertension and diabetes.

Of note, endothelial dysfunction connects with atherosclerosis progression [4], although hyperglycemia and diabetic complications, as metabolic perturbations, influence the endothelial dysfunction in the first step of vascular changes [5] through complex mechanisms including 
oxidative stress (OS) and reactive oxygen species (ROS) [6]. Oxidative stress and derivatives of reactive oxygen metabolites significantly aggravate in diabetic states $[1,3]$, essential hypertension [7], and hyperlipidemia disorders [8], whereas this pathogenesis relates directly to the biological antioxidant capacity of the body [9]. Additionally, recent studies have shown that oxidative stress negatively regulates the adiponectin gene expression [10]; therefore, its concentration in plasma impacts oxidative stress $[2,11]$.

Adiponectin, an adipokine in the human body [12], serves as a biomarker for the determination of oxidative stress of the body [13]. Adiponectin, upon binding to its receptors, increases oxidation of fatty acids and glucose uptake via activating the peroxisome proliferator-activated receptor-gamma (PPAR- $\gamma$ ) ligand pathway [14], thus possessing potential for the treatment of diabetic complications [15] with vascular disorders [16], whereas PPAR- $\gamma$ on activation directly impacts the adiponectin gene transcription [17].

Thiazolidinedione's derivative, pioglitazone, acts as a PPAR- $\gamma$ agonist [18], therapeutically improving insulin resistance and promoting adipocyte differentiation [19]. In addition, the antihypertensive effect of pioglitazone has been ascribed to a reduction in vascular reactivity in terms of vasodilatory action/properties [20] with an increase in plasma adiponectin concentration through PPAR- $\gamma$ activation [21].

In addition, various antihypertensive agents including $\beta$ blockers, calcium channel blockers, ACE inhibitors, and AT1-antagonists partially mediate their effects by decreasing oxidative stress [22]. There are reports that angiotensin II receptor blockers (ARBs) also possess partial agonistic activity for PPAR- $\gamma$ [23]; therefore, irbesartan increases adiponectin production directly by activating PPAR $-\gamma$, selfregulating its AT1R blocker characteristics [24]. Moreover, long-term ARB treatment causes a reduction in pulse wave velocity (PWV) [25], thus inhibiting arterial stiffness, independently, of their antihypertensive property [26].

Nonetheless, very less is known about the antioxidant potential of adiponectin in the genetic model of spontaneously hypertensive rats (SHRs) with type 1 diabetic states. In light of the above background, we tried to evaluate pharmacodynamically the pathophysiological role of exogenously administered adiponectin with PPAR- $\gamma$ agonists in attenuating oxidative stress and arterial stiffness with a variation in metabolic and systemic factors including blood pressure, glycemia, and hypertriglyceridemia in streptozotocin- (STZ-) induced SHRs employing both in vivo and in vitro parameters. The hypothesis also explored whether a potentiating potential or synergistic effect exists between adiponectin with either partial or full PPAR- $\gamma$ agonists, in alleviating oxidative stress caused by STZ in SHRs. Moreover, the relationships between plasma adiponectin and arterial stiffness using pulse wave velocity (PWV) in type 1 diabetic SHRs were also investigated.

\section{Material and Methods}

2.1. Animal Grouping and Experimental Protocol. Eight groups of rats were used for this study $(n=6)$. Forty-two spontaneously hypertensive rats (SHR) and six Wistar Kyoto rats (WKY), averaging 230-255 g body weight, divided randomly into eight groups, were kept in stainless, metabolic cages for 3 days for acclimatization purposes and were fed with commercial rat chow (Gold Coin Sdn. Bhd., Penang, Malaysia) with tap water ad libitum in the animal care facility, Universiti Sains Malaysia, Malaysia (where $n=6$ in each cage), in which six (06) WKYs were used as the control group (WKY+CNT). Forty-two (42) SHRs were divided into 7 groups (where $n=6$ in each group), whereas thirty-six (36) SHRs (6 groups) received STZ and were treated as per experimental protocol and six (6) SHRs served as the control $(\mathrm{SHR}+\mathrm{CNT})$ group. All procedures and animal handling were carried out in accordance with the guidelines research centre "Animal Research and Service Centre (ARASC), USM (main campus)," with ethical approval number: 2012 [28] (352) by the "Animal Ethics Committee, Universiti Sains Malaysia (AECUSM), Malaysia." The animals used in the experiment were grouped following the treatment protocol:

(1) Wistar Kyoto rats: treated with vehicle (WKY+CNT)

(2) SHR: treated with vehicle $(\mathrm{SHR}+\mathrm{CNT})$

(3) SHR+STZ: SHR treated with streptozotocin serving as the SHR diabetic model

(4) SHR+STZ+Irb: given irbesartan $(30 \mathrm{mg} / \mathrm{kg})$ by oral gavage for 28 days starting from day 1

(5) $\mathrm{SHR}+\mathrm{STZ}+\mathrm{Pio}$ : given pioglitazone $(10 \mathrm{mg} / \mathrm{kg})$ orally for 28 days starting from day 1

(6) $\mathrm{SHR}+\mathrm{STZ}+\mathrm{Adp}$ : given adiponectin $2.5 \mu \mathrm{g} / \mathrm{kg} /$ day, intraperitoneal, from day 21 to day 28

(7) $\mathrm{SHR}+\mathrm{STZ}+\mathrm{Irb}+\mathrm{Adp}$ : given irbesartan $(30 \mathrm{mg} / \mathrm{kg})$ by oral gavage for 28 days starting from day 1 and adiponectin $2.5 \mu \mathrm{g} / \mathrm{kg} / \mathrm{day}$, intraperitoneal, from day 21 to day 28

(8) SHR+STZ+Pio+Adp: given pioglitazone $(10 \mathrm{mg} / \mathrm{kg})$ by oral gavage for 28 days starting from day 1 and adiponectin $2.5 \mu \mathrm{g} / \mathrm{kg} / \mathrm{day}$, intraperitoneal, from day 21 to day 28

We prepared a model of type 1 diabetic SHRs using a single intraperitoneal injection (I/P) of (STZ) (Nova Laboratories, Sdn, Bhd, Malaysia), $40 \mathrm{mg} / \mathrm{kg}$ body weight, dissolved in citrate buffer $(10 \mathrm{mM}, \mathrm{pH} 4.5)$ [28], whereas all the STZinduced SHRs were given glucose (10\%) for the first 48 hours after injection to offset the early hypoglycemic shock. Blood glucose levels were evaluated using a standard glucometer (FreeStyle, Abbott, Malaysia), and rats with glucose levels $>300 \mathrm{mg} / \mathrm{dL}$ on the 7 th day were selected for the experiment. Physiological and metabolic perturbations include body weight, $24 \mathrm{hr}$ water intake, and urine collection and were performed on day 0 , to establish the basal variables, followed on days 08,21 , and 28 of the experiment. Systemic hemodynamic parameters including systolic blood pressure (SBP), diastolic blood pressure (DBP), mean arterial pressure (MAP), and heart rate (HR) were measured 
noninvasively (NIBP) using the CODA equipment (Kent Scientific Corporation, Torrington, CT) on a similar day pattern as for metabolic and physiological indices. Pulse wave velocity (PWV) was measured on the acute study day, i.e., day 28. Urine and blood samples were obtained on similar days' pattern, i.e., days $0,8,21$, and 28 of the study. The blood samples $(2 \mathrm{~mL})$ were collected from the tail vein using a rat restrainer; however, plasma samples were obtained following centrifugation of blood at $3500 \mathrm{rpm}$ for 10 minutes and stored at $-30^{\circ} \mathrm{C}$ for further biochemical analysis for parameters including oxidative and antioxidant biomarkers, plasma levels of cholesterol, triglycerides, and low- and high-density lipoprotein measured values. The estimation of plasma adiponectin concentration was carried out using a quantitative assay max rat adiponectin Elisa kit (Chemtron, Biotechnology Sdn, Malaysia).

\subsection{Drugs Used in the Experimental Protocol}

(1) Pioglitazone, ( \pm )-5[4[2(5-ethyl-2-pyridyl)ethoxy]benzyl]-thiazolidine-2,4-dione monohydrochloride (Searle, Pvt, Ltd., Pakistan)

(2) Streptozotocin (STZ, Nova Laboratories, Sdn, Bhd., Selangor, Malaysia)

(3) Irbesartan (Approvel, Sanofi, Aventis, France)

(4) Adiponectin (Chemtron Biotechnology Sdn, Bhd, Malaysia)

A stock solution of pioglitazone $(10 \mathrm{mg} / \mathrm{mL})$ and irbesar$\tan (30 \mathrm{mg} / \mathrm{mL})$ was prepared by dissolving their tablets in distilled water, whereas full-length recombinant adiponectin was dissolved in $200 \mu \mathrm{L}$ phosphate buffer saline [29].

2.3. Measurement of In Vivo Oxidative Stress and Antioxidant Markers. The collected blood plasma samples before the termination of the acute experiment were subjected to a variety of biochemical analyses in order to access the oxidative and antioxidative status of experimental diabetic SHRs. The levels of plasma oxidative stress biomarkers including malondialdehyde (MDA), antioxidant enzyme activities, i.e., total superoxide dismutase (SOD), nitric oxide (NO), total antioxidative activity (TAC), and glutathione peroxidase (GSH) in collected plasma samples were measured using the spectrophotometric detection kits following the instruction manual provided by Institute of Biological Engineering of Nanjing Jiancheng, Nanjing, China.

2.4. Plasma Malondialdehyde. In the biological system, oxygen free radicals can be generated by enzymatic and nonenzymatic reactions. Oxygen free radicals upon generation react with polyunsaturated fatty acids resulting in lipid peroxidation and generate lipid peroxide such as the aldehyde group (malondialdehyde MDA) and ketone and hydroxyl groups with some oxygen free radicals. MDA, a product of lipid peroxidation reactions, is generated as a result of the reaction between free radicals and polyunsaturated fatty acids in the cell membrane [30]. Therefore, evaluation of the MDA concentration in the biological samples could reflect the extent of lipid peroxidation and indirectly signify the extent of cell oxidative state.

2.5. Total Superoxide Dismutase. Superoxide dismutase (SOD) plays an important role in cellular environments in the prevention of diseases linked to oxidative stress. Superoxide dismutase (SOD) scavenges the superoxide anion free radicals and protects the cells from being injured from oxidative stress in a biological system. We investigated SOD measurement in blood plasma samples using the method as described by Oyanagui [31].

2.6. Nitric Oxide. The universal inter- and intracellular molecule, nitric oxide (NO), is involved in regulating the pathophysiology of CVS. Its biological activity is recognized as EDRF responsible for vasodilatation. It is a gaseous free biological molecule with a half-life of few seconds or less in vivo, whereas its altered levels are associated with several pathological conditions like hypertension, hypoxia, and diabetes mellitus. The NO detection kit utilizes the nitrate reductase method and provides an accurate and convenient method for the measurement of total nitrate/nitrite concentration in the biological sample.

2.7. Total Antioxidant Capacity. The antioxidant defence consists of enzymatic and nonenzymatic components. The defence system protects the biological system from oxidation through three pathways. Firstly, it eliminates activated oxygen and free radicals, secondly decomposes superoxide to block the oxidation chain, and lastly gets rid of catalytic metal ions [32]. All different antioxidants yield greater protection against attack by nitrogen radicals and reactive oxygen. Hence, total antioxidant capacity (TOC) provides more concise biological information about antioxidant status of an organism compared to that obtained by the measurement of individual components.

2.8. Plasma Glutathione. Glutathione is a naturally occurring tripeptide and is a significant component of the antioxidant system and offers protection against oxidative damage and in the detoxification processes in the cell. Glutathione is mostly present in its reduced form (GSH) than in the oxidized form (GSSG). GSH is a cofactor for antioxidant enzymes participating in detoxication mechanisms, e.g., glutathione peroxidase (GSH), glutathione transferase, dehydroascorbate, and reductase. GSH scavenges hydroxyl radicals $\left(\mathrm{HO}^{-}\right)$and singlet oxygen $\left({ }^{1} \mathrm{O}_{2}\right)$ directly, whereas the cell redox state can be determined by using the ratio between GSSG/GSH [33].

2.9. Measurement of Plasma Cholesterol, Triglycerides, and Lipoprotein (LDL, HDL) Levels. Triglycerides (ester derivative from fatty acids and glycerol) are transported in plasma by lipoproteins, whereas the excess quantity of carbohydrates converts into triglycerides and deposits in the adipose tissue [34]. We employ the phosphate oxidase/peroxidase method using a biochemical analyzer (ChemWell ${ }^{\circledR}$, Awareness Technology, Inc., FL, USA) for the measurement of plasma triglyceride in collected plasma samples. 
2.10. Surgical Intervention for Pulse Wave Velocity (PWV) Measurement. All animals were fasted overnight (12-14 hours) prior to the surgical interventions used for acute surgery. All cannulae and the transducer were filled with heparinized saline $(20.0$ units $/ \mathrm{mL})$. All animals were anaesthetized with an intraperitoneal injection of $60 \mathrm{mg} / \mathrm{kg}$ sodium pentobarbitone (Nembutal ${ }^{\circledR}$, CEVA Sante Animale, Libourne, France). The trachea was cannulated with a PP240 tube to get a clear airway passage to facilitate respiration. The left jugular vein was cannulated with a PP50 cannula, to which a $50 \mathrm{~mL}$ syringe on an infusion pump (Perfusor secura FT $50 \mathrm{~mL}, \mathrm{~B}$. Braun) that delivered normal saline throughout the experiment was attached. The left carotid artery was catheterized with a PP50 tube for the direct measurement of arterial $\mathrm{BP}$ via a pressure transducer (P23 ID Gould, Statham Instruments, USA) coupled to a computerized data acquisition system $\left(\right.$ Powerlab $^{\circledR}, \mathrm{AD}$ Instruments, Australia). A midline abdominal incision was carried out to expose the left kidney, and the whole dissection process was done using an electrical cautery knife, and the abdominal contents were moved with great care to the right to get the clear exposure of the left kidney. The left kidney was exposed via a ventral midline incision, and a laser Doppler probe $\left(\mathrm{OxyFlo}^{\circledR}\right.$ Probe, Oxford Ltd., UK) attached to the Powerlab ${ }^{\circledR}$ system was placed on the dorsal surface of the kidney for the direct observation of renal cortical blood perfusion (RCBP) values throughout the experiment. Additionally, the left iliac artery was catheterized with a PP50 cannula and was advanced through the abdominal aorta lying at the entrance of the renal artery, whereas the PP50 cannula was kept patent via infusing saline at $3 \mathrm{~mL} / \mathrm{hr}$. A time period of 60 minutes was allowed to stabilize the animals after the completion of the surgical protocol. Blood pressure waves from the two pressure transducers were simultaneously imported and displayed on a data acquisition system at a sampling rate of $400 / \mathrm{s}$ for $30 \mathrm{~min}$. The measurement of PWV was done as per our lab technique methodology, described by Swarup et al. [35] and was calculated by dividing the propagation distance $(\boldsymbol{d})$ by propagation time $(\boldsymbol{t})$ and expressed as meters per second.

2.11. Propagation Distance and Time. At the completion of the acute surgical procedure, the animal was sacrificed with an overdose of sodium pentobarbitone $(200 \mathrm{mg} / \mathrm{kg}$ ) (Nembutal $^{\circledR}$, CEVA, France). The full length of the aorta was exposed, and the tip of the two cannulae from the carotid and iliac arteries was identified and marked. A damp silk thread was placed along the contour of the aorta and marked at the tips of the two cannulae, and the distance between these two points was determined. After that, the thread was removed and laid straight for the measurement of the distance between the two marks identified. This pulse wave propagation distance was used to calculate the PWV. The propagation time was determined using a manual "foot to foot" technique. The time consumed by the pulse wave $(t)$ to move from the aortic arch to the abdominal aorta was measured manually by the time delay between the upstrokes (foot) of each pressure wave front. The average of 10 normal consecutive cardiac cycles was used to calculate the propaga- tion time. Any abnormal waveform within the cycles measured was rejected, and the next viable waveform was measured. The manual foot to foot technique is considered a reliable method for determining PWV $[25,35]$. At the termination of the study, all animals used in the experiment were disposed of in accordance with the guidelines of the Animal Ethics Committee of Universiti Sains Malaysia, Malaysia.

2.12. Statistical Analysis. The statistical analysis was performed using GraphPad Prism ${ }^{\circledR}$ version 5.00 for Windows (GraphPad Software, San Diego, California, USA). Metabolic parameters including body weight, blood glucose level, and plasma adiponectin concentration and the hemodynamics parameters during the treatment period were analyzed using repeated measures one-way ANOVA followed by the Bonferroni post hoc test. Data expressed as the mean \pm SEM and differences between the means were considered significant at the $5 \%$ level.

\section{Results}

3.1. Biochemical and Metabolic Indices. The mean values for metabolic indices including body weight, fluid intake, urine output, and blood glucose concentration of all eight experimental groups were measured on four occasions during the study period, i.e., on day 0 , day 8 , day 21 , and day 28 (Table 1). The initial body weight did not significantly change among all groups including WKY and SHR controls on all four days of observation $(\boldsymbol{P}>\mathbf{0 . 0 5}$. $)$ However, the respective body weights of the control groups (WKY+CNT, SHR+CNT) significantly increased on days 8,21 , and 28 as compared to day $0(\boldsymbol{P}<\mathbf{0 . 0 5})$. As the study progressed, the body weight of the SHR diabetic control $(\mathrm{SHR}+\mathrm{STZ})$ and SHR diabetic treated groups including $\mathrm{SHR}+\mathrm{STZ}+\mathrm{Irb}$, SHR+STZ+Pio, SHR+STZ+Adp, SHR+STZ+Adp+Irb, and SHR+STZ+Adp+Pio follows a significantly decreasing body weight pattern with the duration of diabetes, irrespective of various treatments as compared to day 0 and control groups on days 8,21 , and 28 of the study $(\boldsymbol{P}<\mathbf{0 . 0 5})$ (Table 1).

There was no significant difference in fluid intake in the WKY+CNT group $(\boldsymbol{P}>\mathbf{0 . 0 5})$, but it remained significantly lower in the SHR+CNT group as compared to the WKY control group on all 4 days of observation $(\boldsymbol{P}<\mathbf{0 . 0 5})$. However, in the STZ-induced diabetic model, the SHR+STZ group showed higher water intake on days 8,21 , and 28 as compared to day 0 . Similarly, SHR diabetic treated groups exhibited polydipsia as compared to the SHR+CNT group on days 8,21 , and $28(\boldsymbol{P}<\mathbf{0 . 0 5})$. No significant difference was observed in separate and combined treatment of adiponectin with either irbesartan or pioglitazone $(\mathrm{SHR}+\mathrm{STZ}+$ Adp, SHR+STZ+Adp+Irb, and SHR+STZ+Adp+Pio) on respective days as compared to SHR+STZ $(\boldsymbol{P}>\mathbf{0 . 0 5})$ (Table 1).

Similarly, mean values of the urine flow rate of all experimental groups were observed which was significantly lower in the SHR+CNT as compared to the WKY+CNT group on all 4 days of observation. Contrary to the SHR+CNT group, SHR+STZ-treated rats showed polyuria on days 8,21 , and 
TABLE 1: Metabolic parameters of WKY, SHR control, and SHR diabetic treated groups with irbesartan, pioglitazone, adiponectin, and a combination of adiponectin with irbesartan or pioglitazone.

\begin{tabular}{|c|c|c|c|c|c|}
\hline \multirow{2}{*}{ Parameters } & \multirow{2}{*}{ Groups } & \multicolumn{4}{|c|}{ Days of observation } \\
\hline & & Day 0 & Day 8 & Day 21 & Day 28 \\
\hline \multirow{8}{*}{ Body weight (g) } & $\mathrm{WKY}+\mathrm{CNT}$ & $245 \pm 5$ & $250 \pm 7$ & $275 \pm 4^{*}$ & $289 \pm 8^{*}$ \\
\hline & $\mathrm{SHR}+\mathrm{CNT}$ & $242 \pm 3$ & $248 \pm 6^{\wedge}$ & $267 \pm 8^{*}$ & $284 \pm 9^{*}$ \\
\hline & $\mathrm{SHR}+\mathrm{STZ}$ & $245 \pm 3$ & $200 \pm 5^{*^{\wedge}}$ & $208 \pm 7^{*^{\wedge}}$ & $209 \pm 10^{*}$ \\
\hline & SHR+STZ+Irb & $250 \pm 5$ & $215 \pm 3^{*}$ & $211 \pm 8^{*}$ & $212 \pm 6^{*}$ \\
\hline & SHR+STZ+Pio & $254 \pm 6$ & $205 \pm 4^{*}$ & $207 \pm 7^{*}$ & $217 \pm 5^{* ж}$ \\
\hline & $\mathrm{SHR}+\mathrm{STZ}+\mathrm{Adp}$ & $252 \pm 4$ & $213 \pm 7^{*}$ & $215 \pm 9^{*}$ & $206 \pm 4^{*}$ \\
\hline & SHR+STZ+Irb+Adp & $251 \pm 7$ & $208 \pm 3^{*}$ & $206 \pm 8^{*}$ & $204 \pm 6^{*}$ \\
\hline & $\mathrm{SHR}+\mathrm{STZ}+\mathrm{Pio}+\mathrm{Adp}$ & $247 \pm 5$ & $201 \pm 9^{*}$ & $200 \pm 10^{*}$ & $209 \pm 5^{*}$ \\
\hline \multirow{8}{*}{ Water intake $(\mathrm{mL} / \mathrm{d})$} & $\mathrm{WKY}+\mathrm{CNT}$ & $43 \pm 1$ & $44 \pm 2$ & $45 \pm 3$ & $44 \pm 2$ \\
\hline & $\mathrm{SHR}+\mathrm{CNT}$ & $32 \pm 2^{\wedge}$ & $34 \pm 2^{!}$ & $34 \pm 3^{!}$ & $37 \pm 4^{!}$ \\
\hline & $\mathrm{SHR}+\mathrm{STZ}$ & $33 \pm 2$ & $48 \pm 3^{\wedge *}$ & $48 \pm 2^{\wedge} *$ & $59 \pm 3^{\wedge} *$ \\
\hline & SHR+STZ+Irb & $34 \pm 2$ & $48 \pm 3^{*}$ & $50 \pm 2^{*}$ & $50 \pm 3^{*}$ \\
\hline & SHR+STZ+Pio & $35 \pm 2$ & $47 \pm 2^{*}$ & $51 \pm 2^{*}$ & $60 \pm 3^{*}$ \\
\hline & SHR+STZ+Adp & $36 \pm 2$ & $46 \pm 3^{*}$ & $47 \pm 2^{*}$ & $57 \pm 3^{*}$ \\
\hline & SHR+STZ+Irb+Adp & $35 \pm 2$ & $47 \pm 2^{*}$ & $49 \pm 2^{*}$ & $56 \pm 3^{*}$ \\
\hline & $\mathrm{SHR}+\mathrm{STZ}+\mathrm{Pio}+\mathrm{Adp}$ & $34 \pm 2$ & $45 \pm 3^{*}$ & $49 \pm 2^{*}$ & $55 \pm 3^{*}$ \\
\hline \multirow{8}{*}{ UFR (mL/min/100 g) } & $\mathrm{WKY}+\mathrm{CNT}$ & $3.84 \pm 0.44$ & $3.63 \pm 0.21$ & $3.92 \pm 0.21$ & $3.95 \pm 0.21$ \\
\hline & $\mathrm{SHR}+\mathrm{CNT}$ & $3.10 \pm 0.05^{!}$ & $2.98 \pm 0.09^{!}$ & $2.94 \pm 0.54 !$ & $2.93 \pm 0.43^{!}$ \\
\hline & SHR+STZ & $3.09 \pm 0.04$ & $12.35 \pm 0.52^{\wedge}$ & $12.79 \pm 0.62^{\wedge}$ & $13.18 \pm 0.04^{\wedge}$ \\
\hline & SHR+STZ+Irb & $3.06 \pm 0.02$ & $12.57 \pm 0.25^{*}$ & $12.57 \pm 0.37^{\wedge} *$ & $12.58 \pm 0.22^{* \#}$ \\
\hline & SHR+STZ+Pio & $3.07 \pm 0.03$ & $13.58 \pm 0.55^{* \delta}$ & $13.57 \pm 0.27^{* \delta}$ & $13.58 \pm 0.30^{* \delta ж}$ \\
\hline & SHR+STZ+Adp & $3.06 \pm 0.02$ & $13.28 \pm 0.49^{* \delta}$ & $13.35 \pm 0.15^{* \delta}$ & $16.25 \pm 0.13^{* \delta}$ \\
\hline & SHR+STZ+Irb+Adp & $3.08 \pm 0.02$ & $13.57 \pm 0.39^{* \delta}$ & $13.35 \pm 0.25^{* \delta}$ & $17.79 \pm 0.15^{*} \delta \Phi$ \\
\hline & SHR+STZ+Pio+Adp & $3.09 \pm 0.03$ & $13.57 \pm 0.29^{* \delta}$ & $13.58 \pm 0.24^{* \delta}$ & $20.28 \pm 0.29^{*} \delta \zeta$ \\
\hline \multirow{8}{*}{ Blood glucose (mg/dL) } & $\mathrm{WKY}+\mathrm{CNT}$ & $89 \pm 3$ & $88 \pm 2$ & $86 \pm 2$ & $88 \pm 3$ \\
\hline & $\mathrm{SHR}+\mathrm{CNT}$ & $91 \pm 3$ & $90 \pm 2$ & $88 \pm 3$ & $89 \pm 3$ \\
\hline & $\mathrm{SHR}+\mathrm{STZ}$ & $90 \pm 3$ & $460 \pm 18^{* \wedge}$ & $471 \pm 14^{* \wedge}$ & $489 \pm 25^{*^{\wedge}}$ \\
\hline & SHR+STZ+Irb & $89 \pm 4$ & $458 \pm 11^{*}$ & $462 \pm 17^{*}$ & $470 \pm 16^{*}$ \\
\hline & SHR+STZ+Pio & $90 \pm 5$ & $471 \pm 21^{*}$ & $477 \pm 19^{*}$ & $474 \pm 15^{*}$ \\
\hline & SHR+STZ+Adp & $88 \pm 2$ & $465 \pm 19^{*}$ & $462 \pm 18^{*}$ & $484 \pm 27^{*}$ \\
\hline & SHR+STZ+Irb+Adp & $89 \pm 2$ & $488 \pm 10^{*}$ & $484 \pm 16^{*}$ & $479 \pm 19^{*}$ \\
\hline & SHR+STZ+Pio+Adp & $86 \pm 3$ & $479 \pm 21^{*}$ & $486 \pm 18^{*}$ & $480 \pm 22^{*}$ \\
\hline
\end{tabular}

Notes: the values are presented as the mean $\pm \operatorname{SEM}(n=6)$ in each group and were analyzed by repeated measures one-way ANOVA followed by Bonferroni post hoc test. Values with $P<0.05$ were statistically significant during and at the end of treatment. ! indicates significant difference $(P<0.05)$ between the SHR and WKY control groups. ^ indicates significant difference $(P<0.05)$ between the WKY and SHR control groups in comparison to the SHR diabetic control group. * indicates significant difference $(P<0.05)$ in comparison to day 0 of the respective group. $\delta$ indicates significant difference $(P<0.05)$ of the diabetic Irb, Pio, Adp, Irb+Adp, and Pio+Adp groups in comparison to the SHR diabetic control group. \# indicates significant difference $(P<0.05)$ between the diabetic Irb and Adp groups. $*$ indicates significant difference $(P<0.05)$ between the diabetic Pio and Adp groups. $\Phi$ indicates significant difference $(P<0.05)$ of the diabetic Adp group in comparison to the diabetic Irb+Adp group at days 21 and 28. $\zeta$ indicates a significant difference $(P<0.05)$ of the diabetic Adp group in comparison to the diabetic Pio+Adp group at days 21 and 28.

$28(\boldsymbol{P}<\mathbf{0 . 0 5})$. However, the SHR+STZ+Irb and SHR+STZ + Pio groups did not show a significant difference on days 8,21 , and $28(\boldsymbol{P}>\mathbf{0 . 0 5})$, whereas increased significantly in the SHR+STZ+Adp, SHR+STZ+Irb+Adp, and
SHR+STZ+Pio+Adp groups on day 28 only as compared to the $S H R+S T Z$ group and statistically with greater values in $\mathrm{SHR}+\mathrm{STZ}+\mathrm{Pio}+\mathrm{Adp}$ as compared to the SHR+STZ+ADP and SHR+STZ+Irb+Adp groups $(\boldsymbol{P}<\mathbf{0 . 0 5})($ Table 1$)$. 
All STZ-administered animals developed diabetes resulting in a significant rise in blood glucose levels of the SHR + STZ versus SHR+CNT group $(P<0.05)$, whereas no significant difference was observed between the WKY and SHR control groups on all four days $(P>0.05)$. Similarly, the SHR+STZ- and SHR+STZ-treated groups showed a significant increase in blood glucose values on days 8,21 , and 28 as compared to the SHR+CNT group $(P<0.05)$. However, statistically, there was no significant effect on the blood glucose levels with any set of treatments during the experiment $(P>0.05)$ (Table 1).

3.2. Systemic Hemodynamic. As per the study protocol, baseline values and the changes in the systolic blood pressure (SBP), diastolic blood pressure (DBP), mean arterial pressure (MAP), and heart rate (HR) of eight groups of experimental rats were measured by the tail cuff method on days $0,8,21$, and 28 of the study (Table 2). We observed that SBP and MAP were significantly higher in SHR+CNT as compared to WKY+CNT on all 4 days of observation $(\boldsymbol{P}<\mathbf{0 . 0 5})$, whereas the $\mathrm{SHR}+\mathrm{STZ}$ groups exhibited increased SBP and MAP values as compared to SHR+CNT on days 21 and 28 only $(\boldsymbol{P}>\mathbf{0 . 0 5})$. The SHR+STZ+Irb and $\mathrm{SHR}+\mathrm{STZ}+\mathrm{Pio}$ groups showed a significant decrease in SBP and MAP values on days 21 and 28 and SHR+STZ + Adp on day 28 only as compared to the SHR+STZ group $(\boldsymbol{P}<\mathbf{0 . 0 5})$. Interestingly, the $\mathrm{SHR}+\mathrm{STZ}+\mathrm{Irb}+\mathrm{Adp}$ group expressed greater significant reduction in SBP and MAP on day 28 as compared to other treatments used in the study $(\boldsymbol{P}<\mathbf{0 . 0 5})$, and the values obtained were comparable to the WKY+CNT group (Table 2).

In addition, after induction of diabetes, the mean values of DBP were significantly higher in $\mathrm{SHR}+\mathrm{CNT}$ as compared to $\mathrm{WKY}+\mathrm{CNT}(P<0.05)$, but no significant difference was observed in the SHR+CNT and SHR+STZ groups $(P>0.05$ ) on similar days of observation, whereas a significant decrease in DBP was observed in the SHR+STZ+Irb and $\mathrm{SHR}+\mathrm{STZ}+\mathrm{Pio}$ groups on day 21 and SHR+STZ+Adp on day 28 only as compared to the $\mathrm{SHR}+\mathrm{STZ}+\mathrm{CNT}$ group $(P<0.05)$. Furthermore, DBP of the SHR+STZ+Irb, SHR+STZ+Pio, SHR+STZ+Adp, SHR+STZ+Irb+Adp, and $\mathrm{SHR}+\mathrm{STZ}+\mathrm{Pio}+\mathrm{Adp}$ groups significantly further decrease on day $28(P<0.05)$, with a greater extent of reduction in the SHR+STZ+Irb+Adp group in comparison to the $\mathrm{SHR}+\mathrm{STZ}+\mathrm{Irb}, \mathrm{SHR}+\mathrm{STZ}+\mathrm{Adp}$, and $\mathrm{SHR}+\mathrm{STZ}+\mathrm{Pio}+\mathrm{Adp}$ groups $(P<0.05)$ (Table 2$)$.

The heart rate of all groups was observed on the same pattern of days, i.e., days $0,8,21$, and 28 . The heart rate of the SHR+CNT group remained significantly higher as compared to WKY+CNT on all four points of observation. Moreover, the heart rate of the $\mathrm{SHR}+\mathrm{STZ}+\mathrm{CNT}$ group was significantly higher as compared to $\mathrm{SHR}+\mathrm{CNT}$ on days 21 and $28(\boldsymbol{P}<\mathbf{0 . 0 5})$. However, treating diabetic SHRs significantly reduced the heart rate in $\mathrm{SHR}+\mathrm{STZ}+\mathrm{Irb}, \mathrm{SHR}+\mathrm{STZ}$ + Pio, and SHR+STZ+Adp as compared to SHR+CNT on day $28 \quad(\boldsymbol{P}<\mathbf{0 . 0 5})$, whereas the values obtained in $\mathrm{SHR}+\mathrm{STZ}+\mathrm{Adp}$ were of greater extent as compared to the $\mathrm{SHR}+\mathrm{STZ}+\mathrm{Irb}$ and SHR+STZ+Pio groups. No significant effect was observed in the case of combined treatment of adi- ponectin with either irbesartan or pioglitazone $(\boldsymbol{P}>\mathbf{0 . 0 5})$ (Table 2).

\subsection{Plasma Adiponectin and Lipid Profile Determination.} Plasma adiponectin concentration and lipid profile were measured on day 28 only in the SHR and SHR diabetic pretreated groups. A significant decrease in plasma adiponectin was observed in $\mathrm{SHR}+\mathrm{STZ}$ as compared to the WKY+CNT and SHR+CNT groups $(\boldsymbol{P}<\mathbf{0 . 0 5})$. The diabetic SHRs treated with irbesartan $(30 \mathrm{mg} / \mathrm{kg} /$ day $)$ pioglitazone (10 mg/kg/day), and adiponectin $(2.5 \mu \mathrm{g} / \mathrm{kg} /$ day $)$ expressed a significant increase in plasma adiponectin concentration as compared to the SHR+STZ+CNT group $(\boldsymbol{P}<\mathbf{0 . 0 5})$. Moreover, the combined treatment of adiponectin in the $\mathrm{SHR}+\mathrm{STZ}+\mathrm{Irb}+\mathrm{Adp}$ and $\mathrm{SHR}+\mathrm{STZ}+\mathrm{Pio}+\mathrm{Adp}$ groups significantly increased plasma concentration of adiponectin as compared to their separate treatments $(\boldsymbol{P}<\mathbf{0 . 0 5})$; however, a greater extent of increase in $\mathrm{SHR}+\mathrm{STZ}+\mathrm{Pio}+\mathrm{Adp}$ was observed as compared to the $\mathrm{SHR}+\mathrm{STZ}+\mathrm{Irb}+\mathrm{Adp}$ group $(\boldsymbol{P}<\mathbf{0 . 0 5})$ (Figure 1).

As far as the lipid profile of SHR diabetic treated groups is concerned, SHR+STZ showed a significant increase in triglycerides, low-density lipoproteins, and total serum cholesterol and a decrease in high-density lipoproteins as compared to the SHR+CNT group (Table 3$)(\boldsymbol{P}<\mathbf{0 . 0 5})$. Interestingly, adiponectin treatment (SHR+STZ+Adp) caused a significant improvement in all these parameters $(\boldsymbol{P}<\mathbf{0 . 0 5})$, whereas the combination of adiponectin with pioglitazone $(\mathrm{SHR}+\mathrm{STZ}+\mathrm{Pio}+\mathrm{Adp})$ caused a greater and significant decrease in triglycerides, low-density lipoproteins, and total serum cholesterol with increases in high-density lipoproteins as compared to either their separate treatment or combination of adiponectin with irbesartan $(\boldsymbol{P}<\mathbf{0 . 0 5})$, thus improving the lipid contents of diabetic treated SHRs (Table 3).

3.4. Pulse Wave Velocity and Renal Cortical Blood Perfusion. Recordings for the pulse wave velocity (PWV) and renal cortical blood perfusion (RCBP) for groups including SHR control, STZ-induced diabetic SHRS, and SHR diabetic treated groups were determined during the acute surgical intervention. The RCBP in SHR+STZ was lower as compared to the $\mathrm{WKY}+\mathrm{CNT}$ and $\mathrm{SHR}+\mathrm{CNT}$ groups $(\mathbf{1 3 3} \pm \mathbf{1 2}$ vs. 247 \pm 11 and $167 \pm 9 \mathrm{bpu})$, respectively $(\boldsymbol{P}<0.05)$. The SHR diabetic treated groups (SHR+STZ+Irb, SHR+STZ+Pio, and $\mathrm{SHR}+\mathrm{STZ}+\mathrm{Adp}$ ) showed significantly higher RCBP as compared to the SHR+STZ group $(163 \pm 9,166 \pm 12$, and $187 \pm 9$ vs. $133 \pm 12$ bpu $)$, respectively $(P<\mathbf{0 . 0 5})$. Moreover, $\mathrm{RCBP}$ in the SHR+STZ+Adp group was significantly higher as compared to irbesartan and pioglitazone separate treatments but still remained significantly lower as compared to the WKY+CNT group. The combined treatment in SHR+STZ+Pio+Adp further increased RCBP (209 \pm 12 bpu) and was statistically higher as compared to the SHR + STZ+Irb+Adp group $(\mathbf{1 9 4} \pm \mathbf{6}$ bpu $)(\boldsymbol{P}<\mathbf{0 . 0 5})$ (Figure 2).

Moreover, it was observed that the pulse wave velocity (PWV) of SHR+CNT was significantly higher as compared to the WKY+CNT group, whereas PWV of SHR+STZ was significantly higher compared to the $\mathrm{SHR}+\mathrm{CNT}$ group. This 
TABLE 2: Systemic hemodynamic parameters of WKY, SHR control, and SHR diabetic treated groups with irbesartan, pioglitazone, adiponectin, and a combination of adiponectin with irbesartan or pioglitazone.

\begin{tabular}{|c|c|c|c|c|c|}
\hline \multirow{2}{*}{ Parameters } & \multirow{2}{*}{ Groups } & \multicolumn{4}{|c|}{ Days of observation } \\
\hline & & Day 0 & Day 8 & Day 21 & Day 28 \\
\hline \multirow{8}{*}{ Systolic blood pressure (mmHg) } & $\mathrm{WKY}+\mathrm{CNT}$ & $118 \pm 5$ & $117 \pm 2$ & $117 \pm 2$ & $120 \pm 3$ \\
\hline & $\mathrm{SHR}+\mathrm{CNT}$ & $159 \pm 4^{!}$ & $164 \pm 6^{!}$ & $162 \pm 3 !$ & $157 \pm 8^{!}$ \\
\hline & $\mathrm{SHR}+\mathrm{STZ}$ & $161 \pm 5^{\wedge}$ & $173 \pm 3^{\wedge *}$ & $177 \pm 4^{\wedge} *$ & $175 \pm 3^{\wedge *}$ \\
\hline & $\mathrm{SHR}+\mathrm{STZ}+\mathrm{Irb}$ & $163 \pm 4$ & $177 \pm 5^{*}$ & $147 \pm 4^{* \delta}$ & $135 \pm 3^{* \delta}$ \\
\hline & $\mathrm{SHR}+\mathrm{STZ}+\mathrm{Pio}$ & $165 \pm 6$ & $179 \pm 4^{*}$ & $155 \pm 3^{* \delta ж}$ & $148 \pm 6^{* \delta ж}$ \\
\hline & SHR+STZ+Adp & $162 \pm 4$ & $175 \pm 2^{*}$ & $174 \pm 3^{* \#}$ & $138 \pm 4^{* \delta}$ \\
\hline & SHR+STZ+Irb+Adp & $164 \pm 3$ & $176 \pm 3^{*}$ & $148 \pm 4^{* \delta}$ & $118 \pm 3^{\delta \Phi}$ \\
\hline & SHR+STZ+Pio+Adp & $163 \pm 5$ & $177 \pm 4^{*}$ & $154 \pm 4^{* \delta}$ & $134 \pm 3^{* \delta}$ \\
\hline \multirow{8}{*}{ Diastolic blood pressure (mmHg) } & $\mathrm{WKY}+\mathrm{CNT}$ & $79 \pm 3$ & $84 \pm 4$ & $80 \pm 3$ & $86 \pm 5$ \\
\hline & $\mathrm{SHR}+\mathrm{CNT}$ & $119 \pm 6^{!}$ & $117 \pm 7^{!}$ & $108 \pm 7^{!}$ & $120 \pm 6 !$ \\
\hline & $\mathrm{SHR}+\mathrm{STZ}$ & $117 \pm 3$ & $119 \pm 2$ & $120 \pm 3$ & $118 \pm 4$ \\
\hline & $\mathrm{SHR}+\mathrm{STZ}+\mathrm{Irb}$ & $118 \pm 2$ & $121 \pm 3$ & $101 \pm 2^{* \delta}$ & $92 \pm 1^{* \delta}$ \\
\hline & SHR+STZ+Pio & $116 \pm 3$ & $119 \pm 3$ & $109 \pm 5^{* \delta}$ & $106 \pm 4^{*}$ \\
\hline & SHR+STZ+Adp & $117 \pm 4$ & $120 \pm 4$ & $118 \pm 4^{\# ж}$ & $96 \pm 2^{* \delta \# ж}$ \\
\hline & $\mathrm{SHR}+\mathrm{STZ}+\mathrm{Irb}+\mathrm{Adp}$ & $115 \pm 4$ & $118 \pm 3$ & $103 \pm 3^{* \delta}$ & $88 \pm 2^{* \delta \Phi}$ \\
\hline & SHR+STZ + Pio+Adp & $118 \pm 3$ & $120 \pm 2$ & $111 \pm 3^{* \delta}$ & $98 \pm 2^{* \delta}$ \\
\hline \multirow{8}{*}{ Mean arterial pressure } & $\mathrm{WKY}+\mathrm{CNT}$ & $92 \pm 8$ & $97 \pm 4$ & $92 \pm 4$ & $97 \pm 5$ \\
\hline & $\mathrm{SHR}+\mathrm{CNT}$ & $132 \pm 5^{!}$ & $133 \pm 4^{!}$ & $126 \pm 5^{!}$ & $132 \pm 6^{!}$ \\
\hline & $\mathrm{SHR}+\mathrm{STZ}$ & $132 \pm 7$ & $137 \pm 5^{\wedge} *$ & $143 \pm 4^{\wedge} *$ & $144 \pm 5^{\wedge} *$ \\
\hline & SHR+STZ+Irb & $133 \pm 5$ & $140 \pm 6^{*}$ & $118 \pm 3^{* \delta}$ & $106 \pm 4^{* \delta}$ \\
\hline & $\mathrm{SHR}+\mathrm{STZ}+\mathrm{Pio}$ & $132 \pm 4$ & $139 \pm 4^{*}$ & $124 \pm 4^{* \delta}$ & $120 \pm 5^{* \delta ж}$ \\
\hline & SHR+STZ+Adp & $132 \pm 3$ & $138 \pm 7^{*}$ & $137 \pm 6^{*}$ & $110 \pm 8^{* \delta}$ \\
\hline & SHR+STZ+Irb+Adp & $131 \pm 4$ & $137 \pm 5^{*}$ & $118 \pm 4^{* \delta}$ & $98 \pm 5^{\delta \Phi}$ \\
\hline & SHR+STZ+Pio+Adp & $133 \pm 5$ & $139 \pm 5^{*}$ & $125 \pm 3^{* \delta}$ & $105 \pm 6^{* \delta}$ \\
\hline \multirow{8}{*}{ Heart rate (BPM) } & $\mathrm{WKY}+\mathrm{CNT}$ & $312 \pm 10$ & $310 \pm 4$ & $309 \pm 11$ & $303 \pm 8$ \\
\hline & $\mathrm{SHR}+\mathrm{CNT}$ & $386 \pm 9^{!}$ & $390 \pm 10^{!}$ & $392 \pm 14^{!}$ & $389 \pm 11^{!}$ \\
\hline & $\mathrm{SHR}+\mathrm{STZ}$ & $387 \pm 5^{! \wedge}$ & $394 \pm 7^{\wedge}$ & $402 \pm 4^{\wedge} *$ & $407 \pm 5^{\wedge} *$ \\
\hline & SHR+STZ+Irb & $385 \pm 4$ & $396 \pm 4^{*}$ & $393 \pm 4^{* \delta \#}$ & $386 \pm 3^{\delta \#}$ \\
\hline & $\mathrm{SHR}+\mathrm{STZ}+\mathrm{Pio}$ & $388 \pm 6$ & $400 \pm 3^{*}$ & $380 \pm 3^{* \delta}$ & $367 \pm 4^{* \delta ж}$ \\
\hline & SHR+STZ+Adp & $383 \pm 3$ & $398 \pm 5^{*}$ & $395 \pm 4^{* ж}$ & $356 \pm 3^{* \delta}$ \\
\hline & SHR+STZ+Irb+Adp & $386 \pm 5$ & $401 \pm 4^{*}$ & $396 \pm 3^{* \delta}$ & $360 \pm 4^{* \delta}$ \\
\hline & SHR+STZ+Pio+Adp & $387 \pm 7$ & $403 \pm 5^{*}$ & $377 \pm 6^{* \delta}$ & $351 \pm 5^{* \delta}$ \\
\hline
\end{tabular}

Notes: the values are presented as the mean $\pm \operatorname{SEM}(n=6)$ in each group and were analyzed by repeated measures one-way ANOVA followed by Bonferroni post hoc test. Values with $P<0.05$ were considered statistically significant. ! indicates significant difference $(P<0.05)$ between the SHR and WKY control groups during and at the end of treatment. $*$ indicates significant difference $(P<0.05)$ in comparison to day 0 of the respective group. $\delta$ indicates a significant difference $(P<0.05)$ of the diabetic Irb, Pio, Adp, Irb+Adp, and Pio+Adp groups in comparison to the SHR diabetic control group. \# indicates significant difference $(P<0.05)$ between the diabetic Irb and Adp groups during and at the end of treatment. ж indicates significant difference $(P<0.05)$ between the diabetic Pio and Adp groups during and at the end of treatment. $\Phi$ indicates significant difference $(P<0.05)$ of the diabetic Adp group in comparison to the diabetic Irb+Adp group at days 21 and 28.

increase in PWV was blunted in SHR+STZ+Irb $(\mathbf{6 . 1 7} \pm \mathbf{0 . 1 7}$ $\mathrm{m} / \mathrm{s}), \mathrm{SHR}+\mathrm{STZ}+\mathrm{Pio}+\mathrm{Adp}(6.14 \pm \mathbf{0 . 2 1} \mathrm{m} / \mathrm{s})$, and SHR+STZ $+\operatorname{Adp}(5.49 \pm \mathbf{0 . 2 2} \mathrm{m} / \mathrm{s})$. Furthermore, the tendency to decrease PWV in the adiponectin-treated group was more as compared to the separate irbesartan and pioglitazone groups. Adiponectin with pioglitazone in SHR+STZ+Pio+Adp further reduced PWV and reached the level of the WKY+CNT group $(\mathbf{5} .27 \pm \mathbf{0 . 3 1} \mathrm{m} / \mathrm{s})(\boldsymbol{P}<\mathbf{0 . 0 5})$ (Figure 3$)$. 


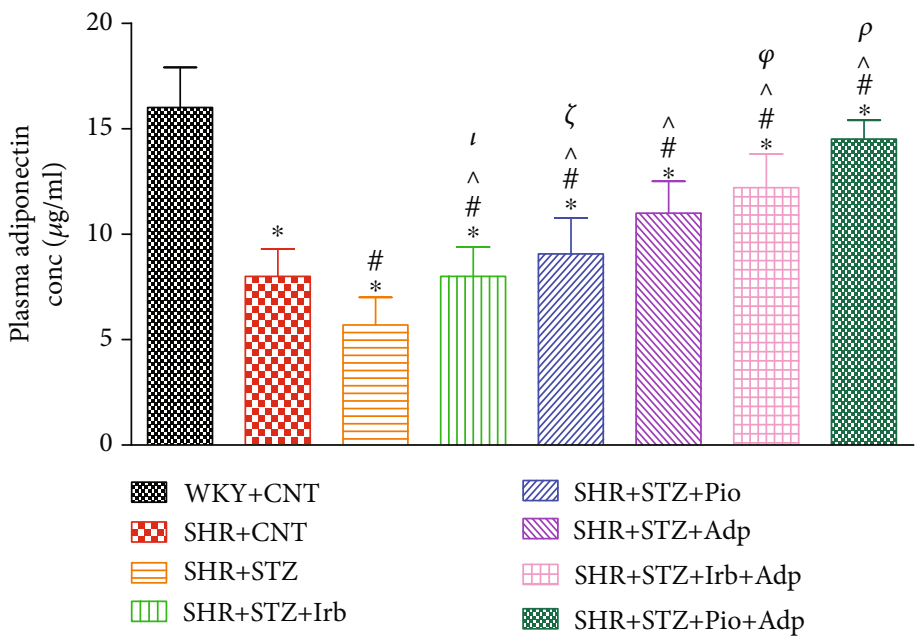

FIGURE 1: Plasma adiponectin concentration in WKY, SHR diabetic control, and SHR diabetic treated rats. The values are presented as the mean \pm SEM $(n=6)$ in each group and were analyzed by one-way ANOVA followed by the Bonferroni post hoc test. Values with $P<0.05$ were considered statistically significant. ${ }^{*} P<0.05$ vs. WKY; ${ }^{\#} P<0.05$ vs. SHR. ${ }^{\wedge} P<0.05$ vs. SHR+STZ+Irb, SHR+STZ+Pio, SHR+STZ+Adp, SHR+STZ+Irb+Adp, and SHR+STZ+Pio+Adp groups in comparison to SHR+STZ. $\tau$ indicates significant difference between SHR+STZ+Irb and SHR+STZ+Adp. $\xi$ indicates significant difference between SHR+STZ+Pio and SHR+STZ+Adp. $\rho$ indicates significant difference between SHR+STZ+Adp and SHR+STZ+Pio+Adp. $\varphi$ indicates significant difference between SHR+STZ+Adp and SHR+STZ+Pio+Adp.

TABLe 3: Plasma triglycerides and lipoprotein (LDL, HDL, and VLDL) level profile of WKY, SHR control, and SHR diabetic treated groups with irbesartan, pioglitazone, adiponectin, and a combination of adiponectin with irbesartan or pioglitazone.

\begin{tabular}{|c|c|c|c|c|}
\hline \multirow{2}{*}{ Groups } & \multicolumn{4}{|c|}{ Lipid profile } \\
\hline & Triglycerides (mg/dL) & Total cholesterol (mg/dL) & HDL (mg/dL) & $\mathrm{LDL}(\mathrm{mg} / \mathrm{dL})$ \\
\hline $\mathrm{WKY}+\mathrm{CNT}$ & $50.75 \pm 4.09$ & $61.25 \pm 2.29$ & $16.33 \pm 1.2$ & $36.25 \pm 1.8$ \\
\hline $\mathrm{SHR}+\mathrm{CNT}$ & $84.80 \pm 11.32 !$ & $149.96 \pm 17.54^{!}$ & $67.64 \pm 3.97^{!}$ & $97.08 \pm 6.33^{!}$ \\
\hline $\mathrm{SHR}+\mathrm{STZ}$ & $172.4 \pm 14.48^{*}$ & $197.72 \pm 12.72^{*}$ & $42.02 \pm 4.63^{*}$ & $122.49 \pm 6.01^{*}$ \\
\hline $\mathrm{SHR}+\mathrm{STZ}+\mathrm{Irb}$ & $153.9 \pm 10.5^{\delta \#}$ & $166.84 \pm 15.0^{\delta \#}$ & $59.78 \pm 4.88^{\delta \#}$ & $108.56 \pm 7.97^{\delta \#}$ \\
\hline $\mathrm{SHR}+\mathrm{STZ}+\mathrm{Pio}$ & $147.2 \pm 10.7^{\delta \varkappa}$ & $153.98 \pm 11.64^{\delta \varkappa}$ & $72.89 \pm 2.85^{\delta \varkappa}$ & $98.96 \pm 7.50^{\delta}$ \\
\hline SHR+STZ+Adp & $93.20 \pm 8.29^{\delta}$ & $129.60 \pm 12.19^{\delta}$ & $79.22 \pm 3.96^{\delta}$ & $96.03 \pm 5.20^{\delta}$ \\
\hline SHR+STZ+Irb+Adp & $85.25 \pm 2.35^{\delta}$ & $136.57 \pm 5.7^{\delta}$ & $79.25 \pm 4.1^{\delta}$ & $95.28 \pm 4.5^{\delta}$ \\
\hline $\mathrm{SHR}+\mathrm{STZ}+\mathrm{Pio}+\mathrm{Adp}$ & $73.25 \pm 4.5^{\delta \zeta}$ & $119.25 \pm 6.7^{\delta \zeta}$ & $77.28 \pm 5.7^{\delta}$ & $91.25 \pm 5.4^{\delta}$ \\
\hline
\end{tabular}

Notes: the values are presented as the mean $\pm \operatorname{SEM}(n=6)$ in each group and were analyzed by one-way ANOVA followed by Bonferroni post hoc test. Values with $P<0.05$ were considered statistically significant during and at the end of treatment. ! indicates significant difference $(P<0.05)$ between the SHR and WKY control groups. * indicates significant difference $(P<0.05)$ in comparison to the SHR control group. $\delta$ indicates significant difference $(P<0.05)$ of the diabetic Irb, Pio, Adp, Irb+Adp, and Pio+Adp groups in comparison to the SHR diabetic control group. \# indicates significant difference $(P<0.05)$ between the diabetic Irb and Adp groups. ж indicates a significant difference $(P<0.05)$ between the diabetic Pio and Adp groups. $\zeta$ indicates a significant difference $(P<0.05)$ of the diabetic Adp group in comparison to the diabetic Pio+Adp group at days 21 and 28.

\subsection{Antioxidant Biomarkers}

3.5.1. Plasma Total Superoxide Dismutase and Malondialdehyde. The plasma total superoxide dismutase (T-SOD) of all experimental groups including diabetic control SHRs and diabetic treated SHRs was measured. The plasma T-SOD of SHR+CNT was significantly lower as compared to WKY+CNT $(\mathbf{1 0 8 . 7 5} \pm \mathbf{3 . 9}$ vs. $145.50 \pm 3.87$ $\mathrm{U} / \mathrm{mL})(\boldsymbol{P}<\mathbf{0 . 0 5})$, whereas STZ+STZ expressed significantly lower values as compared to the $\mathrm{SHR}+\mathrm{CNT}$ group $(\mathbf{1 0 0 . 5 8} \pm \mathbf{4 . 7 7}$ vs. $108.75 \pm 3.9 \mathrm{U} / \mathrm{mL})(\boldsymbol{P}<\mathbf{0 . 0 5})$, which significantly increased in the $\mathrm{SHR}+\mathrm{STZ}+\mathrm{Irb}, \mathrm{SHR}+\mathrm{STZ}+\mathrm{Pio}$, and $\mathrm{SHR}+\mathrm{STZ}+\mathrm{Adp}$ groups as compared to the SHR+STZ group $(119.14 \pm 2.68,125.52 \pm 4.51$, and $138.56 \pm 3.97$ vs. $100.58 \pm 4.77 \mathrm{U} / \mathrm{mL})$, respectively $(\boldsymbol{P}<\mathbf{0 . 0 5})$. The SHR $+\mathrm{STZ}$
+Adp showed significantly higher T-SOD values as compared to the $\mathrm{SHR}+\mathrm{STZ}+\mathrm{Irb}$ and $\mathrm{SHR}+\mathrm{STZ}+\mathrm{Pio}$ groups. The combined treatment of adiponectin with pioglitazone in SHR+STZ+Pio+Adp further increases T-SOD values $(\mathbf{1 4 6 . 2 7} \pm \mathbf{5 . 0 1} \mathrm{U} / \mathrm{mL})$ and reaches the level of $\mathrm{WKY}+\mathrm{CNT}$ $(\boldsymbol{P}>\mathbf{0 . 0 5})$, as compared to the SHR+STZ+Irb+Adp group which did not show a significant difference to the SHR+STZ +Adp group $(\mathbf{1 4 3 . 2 5} \pm \mathbf{3 . 8 1} \mathrm{U} / \mathrm{mL})(\boldsymbol{P}>\mathbf{0 . 0 5})$ (Figure 4).

We also obtained the plasma malondialdehyde (MDA) levels of these experimental groups, which were significantly higher in SHR+CNT as compared to WKY+CNT $(5.91 \pm 0.22$ vs. $2.85 \pm 0.19 \mathrm{nmol} / \mathrm{mL})(\boldsymbol{P}<\mathbf{0 . 0 5})$, whereas plasma MDA levels in SHR+STZ were significantly higher as compared to the SHR+CNT group $(\mathbf{6 . 6 1} \pm \mathbf{0 . 2 5}$ vs. $\mathbf{5 . 9 1}$ $\pm \mathbf{0 . 2 3} \mathrm{nmol} / \mathrm{mL})$. The separate treatments with either 


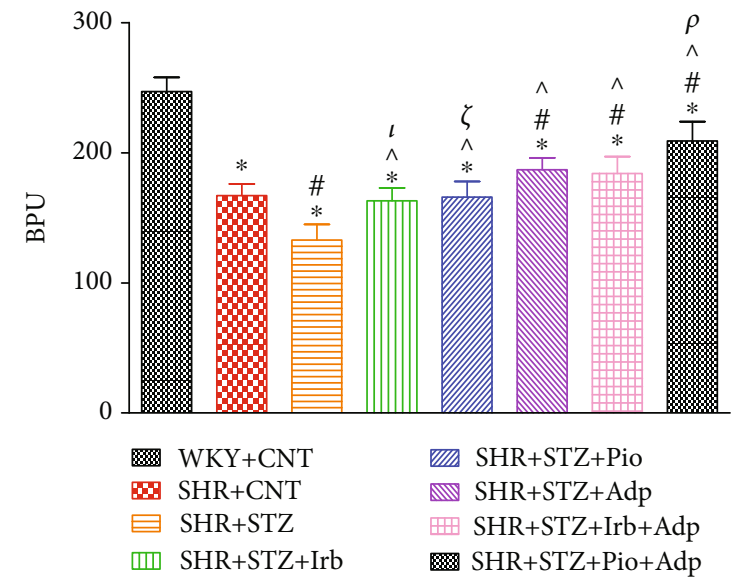

FIGURE 2: Baseline renal cortical blood perfusion of WKY, SHR diabetic control, and SHR diabetic treated rats. The values are presented as the mean \pm SEM $(n=6)$ in each group and were analyzed by one-way ANOVA followed by Bonferroni post hoc test. Values with $P<0.05$ were considered statistically significant. ${ }^{*} P<0.05$ versus WKY; ${ }^{\#} P<0.05$ versus $\mathrm{SHR} ;{ }^{\wedge} P<0.05$ vs. SHR +STZ+Irb, SHR+STZ+Pio, SHR+STZ+Adp, SHR+STZ+Irb+Adp, and $\mathrm{SHR}+\mathrm{STZ}+\mathrm{Pio}+\mathrm{Adp}$ groups in comparison to SHR+STZ. $\tau$ indicates significant difference between $\mathrm{SHR}+\mathrm{STZ}+\mathrm{Irb}$ and SHR+STZ+Adp. $\xi$ indicates significant difference between $\mathrm{SHR}+\mathrm{STZ}+\mathrm{Pio}$ and $\mathrm{SHR}+\mathrm{STZ}+\mathrm{Adp} . \rho$ indicates significant difference between $\mathrm{SHR}+\mathrm{STZ}+\mathrm{Adp}$ and $\mathrm{SHR}+\mathrm{STZ}+\mathrm{Pio}+\mathrm{Adp}$.

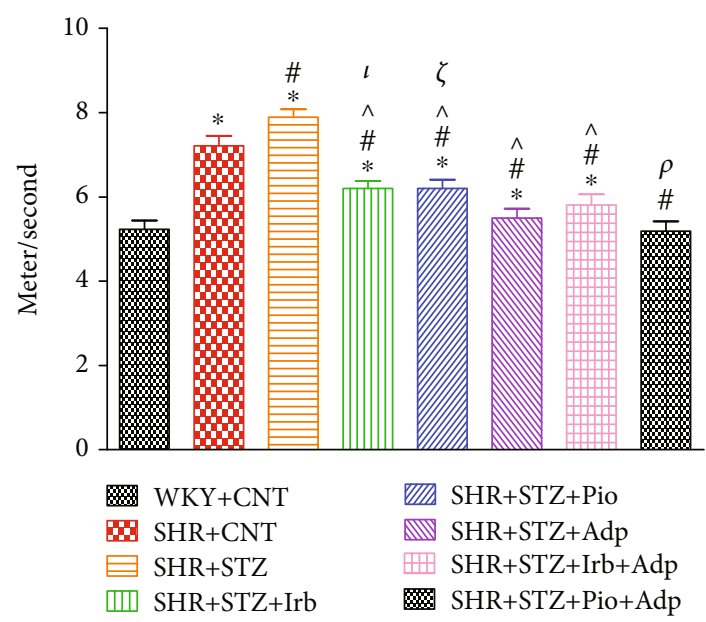

Figure 3: Pulse wave velocity of WKY, SHR diabetic control, and SHR diabetic treated rats. The values are presented as the mean \pm SEM $(n=6)$ in each group and were analyzed by one-way ANOVA followed by Bonferroni post hoc test. Values with $P<$ 0.05 were considered statistically significant. ${ }^{*} P<0.05$ versus WKY; ${ }^{\#} P<0.05$ versus SHR; ${ }^{\wedge} P<0.05$ vs. SHR+STZ+Irb, SHR + STZ+Pio, SHR+STZ+Adp, SHR+STZ+Irb+Adp, and SHR+STZ + Pio+Adp groups in comparison to SHR+STZ. $\tau$ indicates significant difference between $\mathrm{SHR}+\mathrm{STZ}+\mathrm{Irb}$ and $\mathrm{SHR}+\mathrm{STZ}+\mathrm{Adp}$. $\xi$ indicates significant difference between $\mathrm{SHR}+\mathrm{STZ}+\mathrm{Pio}$ and SHR + STZ+Adp. $\rho$ indicates significant difference between SHR+STZ + Adp and SHR+STZ+Pio+Adp.

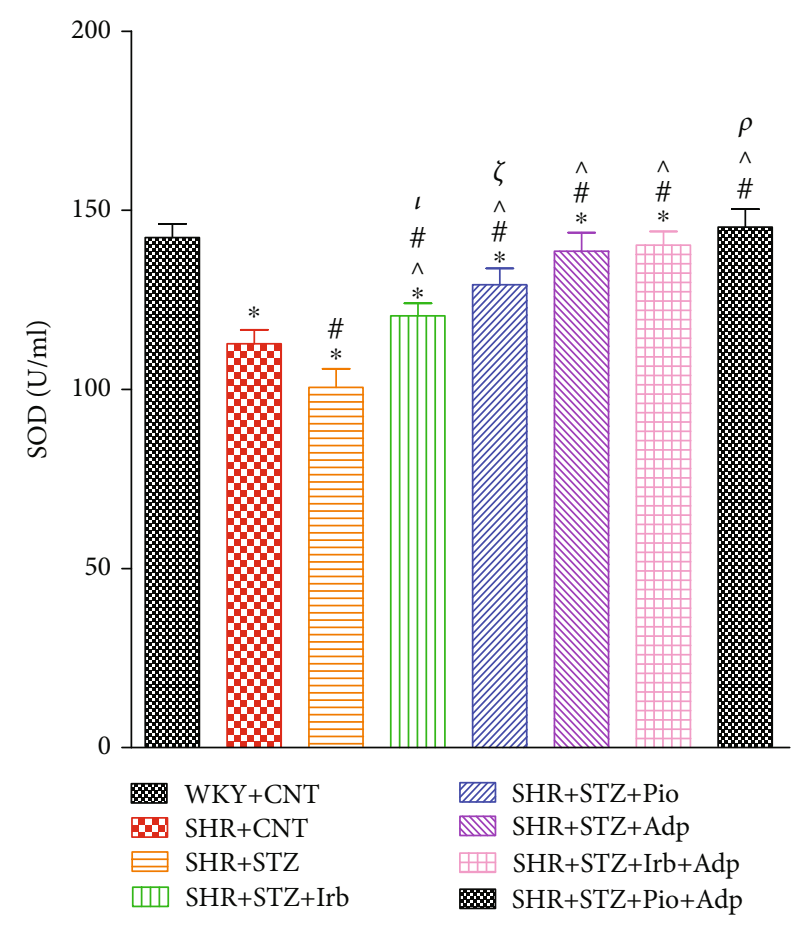

FIgURE 4: Plasma total superoxide dismutase levels of WKY, SHR diabetic control, and SHR diabetic treated rats. The values are presented as the mean \pm SEM $(n=6)$ in each group and were analyzed by one-way ANOVA followed by Bonferroni post hoc test. Values with $P<0.05$ were considered statistically significant. ${ }^{*} P<0.05$ versus WKY; ${ }^{\#} P<0.05$ versus SHR; ${ }^{\wedge} P<0.05$ vs. SHR +STZ+Irb, SHR+STZ+Pio, SHR+STZ+Adp, SHR+STZ+Irb+Adp, and $\mathrm{SHR}+\mathrm{STZ}+\mathrm{Pio}+\mathrm{Adp}$ groups in comparison to SHR+STZ. $\tau$ indicates significant difference between $\mathrm{SHR}+\mathrm{STZ}+\mathrm{Irb}$ and SHR + STZ+Adp. $\xi$ indicates significant difference between SHR+STZ + Pio and SHR+STZ+Adp. $\rho$ indicates significant difference between SHR+STZ+Adp and SHR+STZ+Pio+Adp.

irbesartan or pioglitazone (SHR+STZ+Irb, SHR+STZ+Pio) significantly reduced $\mathrm{MDA}$ concentrations as compared to the SHR+CNT group $(5.25 \pm 0.25$ and $4.99 \pm 0.21$ vs. 6.61 $\pm 0.25 \mathrm{nmol} / \mathrm{ml})$, respectively $(P<\mathbf{0 . 0 5})$. Furthermore, treatment with adiponectin $(\mathrm{SHR}+\mathrm{STZ}+\mathrm{Adp})$ and its combination with the irbesartan $(\mathrm{SHR}+\mathrm{STZ}+\mathrm{Irb}+\mathrm{Adp})$ or pioglitazone (SHR+STZ+Pio+Adp) groups further significantly decreased the plasma MDA concentration $(3.01 \pm 0.17,2.99 \pm 0.11$, and $2.95 \pm 0.01 \mathrm{nmol} / \mathrm{L})$, respectively $(P<0.05)$. There was no significant difference between the SHR+STZ+Adp group as compared to the SHR+STZ+Irb+Adp and SHR+STZ+Pio + Adp groups $(\boldsymbol{P}>\mathbf{0 . 0 5})$ (Figure 5).

\subsubsection{The Plasma Nitric Oxide and Total Antioxidant} Capacity. The plasma nitric oxide (NOx) levels were estimated by measuring the total nitrate/nitrite concentrations in plasma. We observed that the plasma NO level of SHR+CNT was significantly lower as compared to the $\mathrm{WKY}+\mathrm{CNT}$ group $(\mathbf{2 2 . 5 4} \pm \mathbf{0 . 7 7}$ vs. $33.12 \pm \mathbf{0 . 9 7} \mu \mathrm{mol} / \mathrm{L})$, whereas the plasma NO level of SHR+STZ was significantly lower as compared to the SHR $+\mathrm{CNT}$ group $(\mathbf{2 0 . 5 1} \pm \mathbf{0 . 8 6}$ vs. $22.54 \pm 0.77 \mu \mathrm{mol} / \mathrm{L})$, respectively $(\boldsymbol{P}<\mathbf{0 . 0 5})$. Interestingly, pioglitazone and adiponectin single treatments significantly 


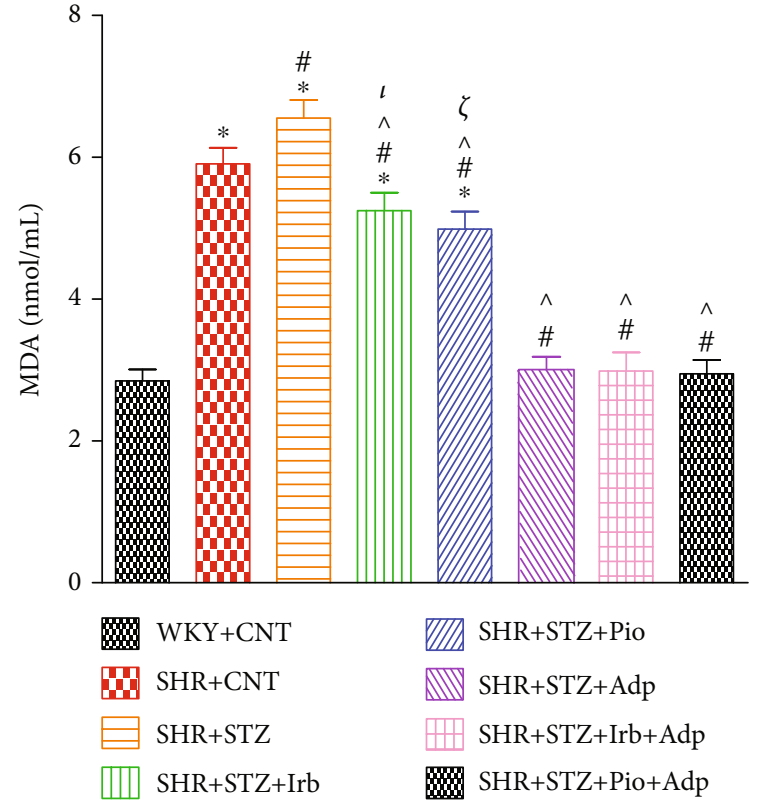

FIgURE 5: Plasma malondialdehyde levels of WKY, SHR diabetic control, and SHR diabetic treated rats. The values are presented as the mean $\pm \operatorname{SEM}(n=6)$ in each group and were analyzed by oneway ANOVA followed by Bonferroni post hoc test. Values with $P<$ 0.05 were considered statistically significant. ${ }^{*} P<0.05$ versus WKY; ${ }^{\#} P<0.05$ versus $S H R ;{ }^{\wedge} P<0.05$ vs. SHR+STZ+Irb, SHR+STZ+Pio, SHR+STZ+Adp, SHR+STZ+Irb+Adp, and SHR+STZ+Pio+Adp groups in comparison to SHR+STZ. $\tau$ indicates significant difference between SHR+STZ+Irb and SHR+STZ+Adp. $\xi$ indicates significant difference between $\mathrm{SHR}+\mathrm{STZ}+\mathrm{Pio}$ and $\mathrm{SHR}+\mathrm{STZ}+\mathrm{Adp}$.

increased plasma NO levels in the SHR+STZ+Pio and SHR+STZ+Adp groups as compared to the SHR+STZ and SHR+STZ+Irb groups $(23.56 \pm \mathbf{0 . 6 5}$ and $28.52 \pm \mathbf{0 . 3 9}$ vs. $20.51 \pm 0.86$ and $21.70 \pm 0.71 \mu \mathrm{mol} / \mathrm{L})$, respectively $(\boldsymbol{P}<\mathbf{0 . 0 5})$. The combined treatment of adiponectin with pioglitazone (SHR+STZ+Pio+Adp) further increased the plasma NO level $(32.77 \pm \mathbf{0 . 8 8} \mu \mathrm{mol} / \mathrm{L}) \quad(\boldsymbol{P}<\mathbf{0 . 0 5})$ and was comparable to the WKY+CNT group (Figure 6).

Our observations also recorded significantly decreased values for total antioxidant capacity (TAC) in SHR+CNT as compared to the $\mathrm{WKY}+\mathrm{CNT}$ group $(\mathbf{1 . 3 7} \pm \mathbf{0 . 0 9}$ vs. $1.99 \pm 0.05 \mathrm{U} / \mathrm{mL})(\boldsymbol{P}<\mathbf{0 . 0 5})$, whereas the plasma TAC values in SHR+STZ further significantly reduced as compared to the SHR+CNT group $(\mathbf{1 . 1 2} \pm \mathbf{0 . 0 7}$ vs. $\mathbf{1 . 3 7} \pm \mathbf{0 . 0 9}$ $\mathrm{U} / \mathrm{mL})(\boldsymbol{P}<\mathbf{0 . 0 5})$. Moreover, treated SHRs with either irbesartan (SHR+STZ+Irb) or pioglitazone (SHR+STZ+Pio) caused significantly increased TAC values as compared to the SHR+STZ group $(1.33 \pm 0.08$ and $1.39 \pm 0.05$ vs. 1.12 $\pm \mathbf{0 . 0 7} \mathrm{U} / \mathbf{m L})$, respectively $(P<\mathbf{0 . 0 5})$. The plasma TAC values significantly increased in the SHR+STZ+Adp, SHR + STZ+Irb+Adp, and SHR+STZ+Pio+Adp groups $(1.70 \pm 0.09,1.85 \pm 0.11$, and $2.01 \pm 0.07 \mathrm{U} / \mathrm{mL})$, respectively $(\boldsymbol{P}<\mathbf{0 . 0 5})$, with greater values obtained in SHR+STZ+Pio+Adp $(\mathbf{2 . 0 1} \pm \mathbf{0 . 0 7} \mathrm{U} / \mathrm{mL})$, and were comparable to the WKY+CNT group $(\mathbf{1 . 9 9} \pm \mathbf{0 . 0 5} \mathrm{U} / \mathrm{mL})$ $(\boldsymbol{P}<\mathbf{0 . 0 5})$ (Figure 7).

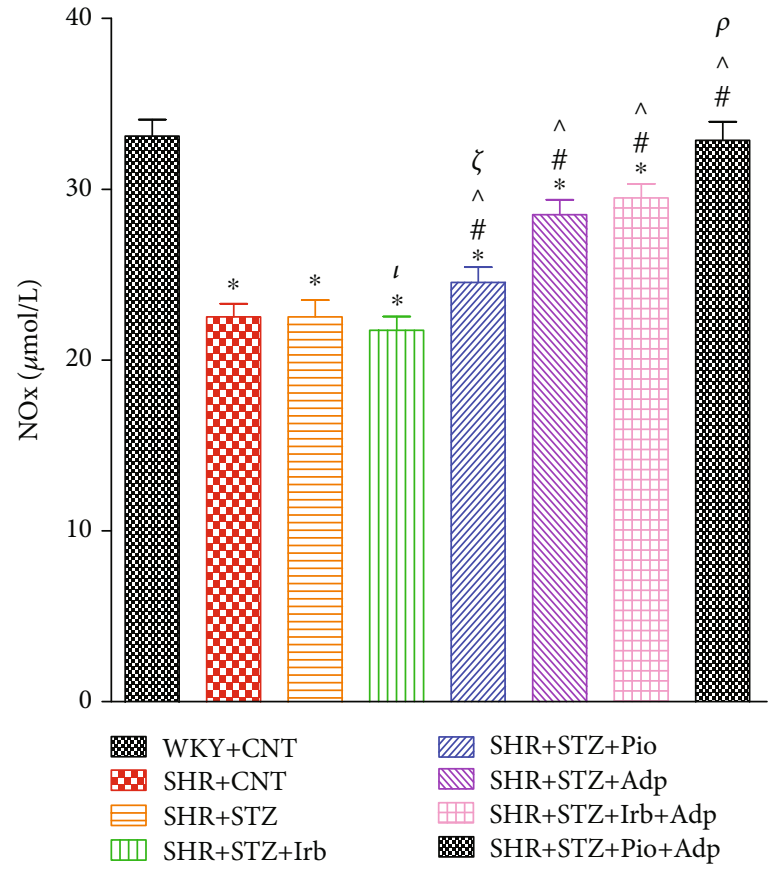

Figure 6: Plasma nitric oxide levels of WKY, SHR diabetic control, and SHR diabetic treated rats. The values are presented as the mean $\pm \operatorname{SEM}(n=6)$ in each group and were analyzed by one-way ANOVA followed by Bonferroni post hoc test. Values with $P<$ 0.05 were considered statistically significant. ${ }^{*} P<0.05$ versus WKY; ${ }^{\#} P<0.05$ versus SHR; ${ }^{\wedge} P<0.05$ vs. SHR+STZ+Irb, SHR + STZ+Pio, SHR+STZ+Adp, SHR+STZ+Irb+Adp, and SHR+STZ + Pio+Adp groups in comparison to SHR+STZ. $\tau$ indicates significant difference between $\mathrm{SHR}+\mathrm{STZ}+\mathrm{Irb}$ and $\mathrm{SHR}+\mathrm{STZ}+\mathrm{Adp}$. $\xi$ indicates significant difference between $\mathrm{SHR}+\mathrm{STZ}+\mathrm{Pio}$ and SHR + STZ+Adp. $\rho$ indicates significant difference between SHR+STZ +Adp and SHR+STZ+Pio+Adp.

3.5.3. Plasma Glutathione. In the last, we also measured plasma glutathione (GSH) in SHR diabetic treated groups, which showed significantly lower values in $\mathrm{SHR}+\mathrm{CNT}$ as compared to the WKY+CNT group $(120.19 \pm 3.85$ vs. $160.08 \pm 4.10 \mu \mathrm{mol} / \mathrm{L}) \quad(P<0.05) \quad$ (Figure 8$)$. However, SHR+STZ significantly reduced GSH values as compared to the SHR+CNT group $(110.23 \pm 3.77$ vs. $120.19 \pm 3.85 \mu$ $\mathrm{mol} / \mathrm{L})(P<0.05)$. Single treatments with irbesartan, pioglitazone, and adiponectin significantly increased GSH values in SHR+STZ+Irb, SHR+STZ+Pio, and SHR+STZ+Adp as compared to the SHR+STZ group $(133.49 \pm 3.77,139.22 \pm 3.66$, and $145.49 \pm 5.13$ vs.110.21 \pm $3.77 \mu \mathrm{mol} / \mathrm{L})$, respectively $(P<0.05)$. The combined treatment of adiponectin with pioglitazone in SHR + STZ+Pio+Adp further increased GSH values $(156.27 \pm 3.77)$ as compared to the SHR+STZ+Irb+Adp group $\quad(150.25 \pm 4.77 \mu \mathrm{mol} / \mathrm{L}) \quad(P<0.05)$ but was not comparable to the WKY control group $(160.08 \pm 4.10 \mu \mathrm{mol} / \mathrm{L})$ (Figure 8).

\section{Discussion}

To the best of our knowledge, this study is among few investigating the pathophysiological role and impact of 


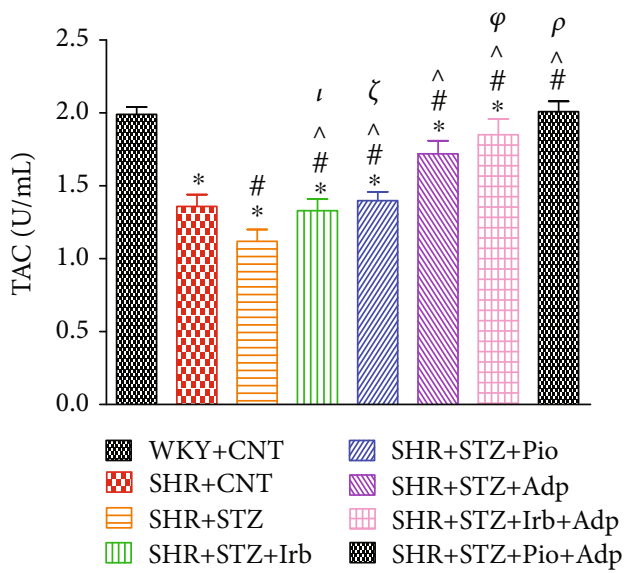

FIGURE 7: Plasma total antioxidant capacity of WKY, SHR diabetic control, and SHR diabetic treated rats. The values are presented as the mean $\pm \operatorname{SEM}(n=6)$ in each group and were analyzed by oneway ANOVA followed by Bonferroni post hoc test. Values with $P$ $<0.05$ were considered statistically significant. ${ }^{*} P<0.05$ versus WKY; ${ }^{\#} P<0.05$ versus SHR; ${ }^{\wedge} P<0.05$ vs. SHR+STZ+Irb, SHR + STZ+Pio, SHR+STZ+Adp, SHR+STZ+Irb+Adp, and SHR+STZ $+\mathrm{Pio}+\mathrm{Adp}$ groups in comparison to SHR+STZ. $\tau$ indicates significant difference between $\mathrm{SHR}+\mathrm{STZ}+\mathrm{Irb}$ and $\mathrm{SHR}+\mathrm{STZ}+\mathrm{Adp}$. $\xi$ indicates significant difference between SHR+STZ+Pio and SHR + STZ+Adp. $\rho$ indicates significant difference between SHR+STZ + Adp and SHR+STZ+Pio+Adp. $\varphi$ indicates significant difference between $\mathrm{SHR}+\mathrm{STZ}+\mathrm{Adp}$ to $\mathrm{SHR}+\mathrm{STZ}+\mathrm{Pio}+\mathrm{Adp}$.

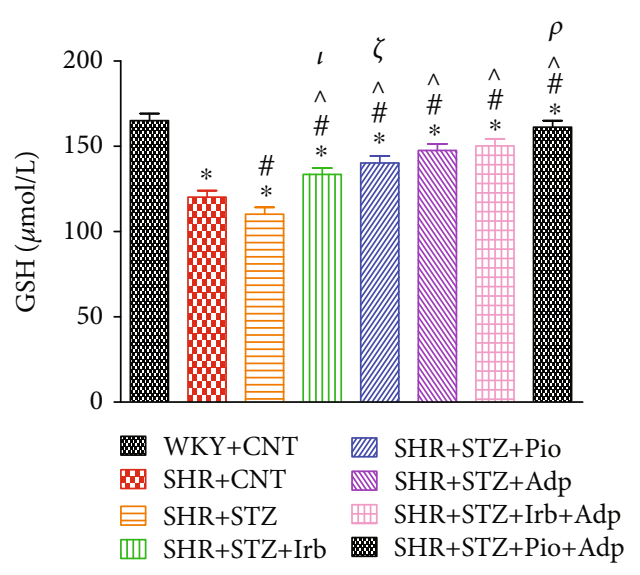

FIGURE 8: Plasma glutathione level of WKY, SHR diabetic control, and SHR diabetic treated rats. The values are presented as the mean $\pm \operatorname{SEM}(n=6)$ in each group and were analyzed by one-way ANOVA followed by Bonferroni post hoc test. Values with $P<$ 0.05 were considered statistically significant. ${ }^{*} P<0.05$ versus WKY; ${ }^{\#} P<0.05$ versus SHR; ${ }^{\wedge} P<0.05$ vs. SHR+STZ+Irb, SHR +STZ+Pio, SHR+STZ+Adp, SHR+STZ+Irb+Adp, and SHR+STZ $+\mathrm{Pio}+\mathrm{Adp}$ groups in comparison to $\mathrm{SHR}+\mathrm{STZ}$. $\tau$ indicates significant difference between $\mathrm{SHR}+\mathrm{STZ}+\mathrm{Irb}$ and $\mathrm{SHR}+\mathrm{STZ}+\mathrm{Adp}$. $\xi$ indicates significant difference between $\mathrm{SHR}+\mathrm{STZ}+\mathrm{Pio}$ and SHR + STZ+Adp. $\rho$ indicates significant difference between SHR+STZ + Adp and SHR+STZ+Pio+Adp. exogenously administered adiponectin with PPAR- $\gamma$ agonists in streptozotocin- (STZ-) induced spontaneously hypertensive rats (SHRs) by measuring in vivo and in vitro antioxidant potential, plasma lipid contents, and glycemic and endogenous adiponectin levels with systemic and renal blood pressure measurements. Our results indicate that the continuous administration of STZ had led to vascular abnormalities and impaired endothelial functions with decreased plasma adiponectin concentration. The present study also assessed the relationship between pulse wave velocity (PWV) and oxidative stress markers. Our study indicates that STZ administration leads to a complex mechanism of diabetes and hypertension development, possibly due to the enhanced oxidative stress, indicated by increased MDA and decreased plasma SOD, NOx, and TOC levels. The 28 days of the study period, pharmacodynamically, revealed that adiponectin, as a biomarker, in combination with full PPAR- $\gamma$ agonist, pioglitazone, abrogates oxidative stress including PWV and ameliorates lipid profile and systemic and renal blood pressure without affecting glycemic levels, signifying the synergistic antioxidant potential and vasodilator action in pretreated diabetic SHRs.

Spontaneously hypertensive rats (SHRs) are more susceptible to diabetogenic effect of streptozotocin, most frequently used for the induction of diabetes [36] and causes increased production of reactive oxygen species (ROS) with activation of polyadenosine diphosphate ribosylation and nitric oxide release in SHRs [37].

Oxidative stress has been demonstrated in the pathogenesis of hypertension in SHR hypertension and diabetes. Vascular oxidative stress has been observed in different models of experimental hypertension like angiotensin II-induced hypertension, Dahl salt-sensitive hypertension, and obesityassociated hypertension and in SHR [38], lead-induced, salt-sensitive, and essential hypertension and diabetes mellitus [7]. Consequently, we attempted to develop a wellknown rat model of a combined state of essential hypertension with diabetes.

Physiological and metabolic indices were kept into consideration to assess experimental diabetes in SHRs, including body weight, which was significantly reduced as one of the pronounced effects of STZ on $\beta$-cells [39]. Polyuria and polydipsia were also observed as significant metabolic perturbations of diabetic SHRs and found to be in accordance with the observations of Khan et al. [40]. As an acceptable explanation for the observed polydipsia among the rats with experimental early diabetic nephropathy as reported by others and us, this could be due to the fluid loss in the face of severe polyuria in these animals [41]. However, SHR $+\mathrm{CNT}$ only showed a decrease in fluid intake as compared to WKY+CNT. This could be due to the species variation, difference in sodium metabolism [42], and an overexpression of Ang-II, aldosterone [43], and vasopressin in this genetic model of hypertensive rats. Another explanation for this could be the decreased plasma concentration of adiponectin in SHRs as compared to normotensive (WKY) which causes an inhibitory effect on ADH secretion and retains fluid volume in the body, thus indirectly leading to a decrease in fluid intake in the nontreated/control group. 
Hyperglycemia due to poor glycemic control is common in overt diabetes and is associated with dysfunctions of different organs, particularly the kidney, nerves, eye, heart, and blood vessels [44]. Hyperglycemia with elevated BP causes damage to the vascular endothelial cells with increased oxidative stress and vascular reactivity [45] and is considered a vital phenomenon of oxidative stress [46]. Diabetes associated with hyperglycemia modifies the endothelial function through a number of complex mechanisms including oxidative stress [47], glycation of protein and lipids [48], and activation of protein kinase C [49]. Similarly, the endothelial-dependent vasodilatation is impaired in different animal models of hypertension including spontaneous hypertensive rats and renovascular hypertension [50]. Therefore, ROS formation can be a direct consequence of hyperglycemia.

In our study, the glycemic level was not influenced after either irbesartan, adiponectin, or pioglitazone either single or combination treatment protocol. This probably corresponds with the type of the diabetic model using STZ similar to the human type 1 diabetic model. It is well known that endothelial dysfunction occurs in diabetic complications [6], associates with atherosclerotic progression [4], and elevates in oxidative stress. In diabetic SHRs, variable observation in terms of increased or decreased SBP and MAP is reported $[51,52]$, whereas in our study findings, the MAP and SBP values of diabetic SHRs were considerably higher, which could be due to the rapid destruction of nitric oxide (NO) in STZ-induced SHRs [53], although diminished NO bioactivity and bioavailability are key characteristics for arterial hypertension and endothelial disorders [54].

Moreover, we also observed that hyperglycemic SHRs exhibited decreased RCBP which supports previous observations of our laboratory findings in a diabetic model of rats $[40,51]$, which is probably due to stimulation of local Ang-II and intrarenal RAAS [55]. In our findings, three weeks of irbesartan (partial PPAR- $\gamma$ agonist) in combination with adiponectin significantly reduced RCBP, SBP, and MAP values to a larger extent as compared to adiponectin and pioglitazone (full PPAR- $\gamma$ agonist) either single or combination pretreatments, which could be possibly due to upregulation of PPAR $-\gamma$ receptors besides an increase in production of nitric oxide (NO) $[56,57]$. The significance of $\mathrm{NO}$ in the kidney vasculature cannot be ruled out which performs various pivotal roles including renal hemodynamic regulation, modulation of renal sympathetic neural activity, and inhibition of the tubular sodium reabsorptive mechanism [58]. We observed that irbesartan at $30 \mathrm{mg} / \mathrm{kg} / \mathrm{day}$ caused a maximal dose for blockade of RAAS while its partial PPAR- $\gamma$ agonistic activity also contributed to its BP reduction and renoprotective characteristics in nondiabetic SHRs as observed previously in our findings [59].

Nonetheless, regulation of MAP and vascular tone depends upon NO, which acts as an endothelium-derived molecule [60], whereas plasma adiponectin stimulates production of NO with reduction in sensitivity to Ang-II [61]. Adiponectin receptors (Adipo R1 and Adipo R2) in endothelial cells mediate adiponectin-induced phosphorylation of AMPK and eNOS which together lead to an increase in
NO production [62]. In our findings, activation of PPAR- $\gamma$ with partial and full agonists (irbesartan and pioglitazone), respectively, upregulates plasma adiponectin levels probably by stimulating the expression of proteins involved in adiponectin assembly, for instance, endoplasmic reticulum oxidoreductin-1 protein (Erol-L $\alpha$ ) and adiponectin secretion such as disulfide-bond A oxidoreductase-like protein (DsbA-L) [63]; however, we did not measure these proteins in our experimental protocol.

Moreover, in our findings, the heart rate of STZ-induced SHRs remained higher which could be due to the SNS overactivation [64], whereas the hypertensive state correlates with SNS activity, which, therefore, is intricately involved with the initiation and progression of hypertension causing increases in the heart rate [65] and supports our values obtained in diabetic SHRs. Previous findings confirm the adiponectin existence in the cerebrospinal fluid [66], thus controlling and reducing the sympathetic nerve activity and heart rate [67], indicating that adiponectin is merely responsible for the reduction in the heart rate of diabetic SHRs treated groups.

4.1. Adiponectin Concentration in Plasma and Lipid Profile. Diabetes induced by high-dose STZ is similar to the human type 1 diabetic model [68]; thus, reduction of plasma adiponectin concentration with STZ administration would contribute to the diabetic condition of SHRs and is in agreement with findings of Thule et al. [69]. Interestingly, in our experimental protocol, STZ-induced SHRs treated with pioglitazone for 3 weeks in combination with exogenous adiponectin significantly increased adiponectin levels as compared to the other sets of treatment. It is also evident that pioglitazone while acting as an agonist for PPAR- $\gamma$ improves endothelial function [70], with BP reduction and lipid metabolism [71] via stimulating the production of plasma adiponectin [21] and reduction in vascular sensitivity in diabetic SHRs [20].

In addition, we also measured lipid contents of experimental diabetic and genetic models of hypertensive pretreated rats. Plasma triglyceride concentrations were higher in control SHRs as compared to control WKY during the treatment period, whereas STZ treatment aggravates the condition in a genetic model of hypertensive rats, leading to a further significant increase in plasma triglyceride, LDL, and total serum cholesterol, with a decrease in adiponectin and HDL plasma concentrations, indicating anthropometric and physiological disorders. Previous studies reveal that full-length adiponectin activates AMP-activated protein kinase (AMPK) phosphorylation [72] stimulating fatty-acid oxidation and glucose utilization by activating AMP-activated protein kinase, thus suppressing gluconeogenesis in the liver [14]. However, phosphorylation of AMPK regulates enzymes responsible for the synthesis of triglycerides and fatty acids with their transcription factors, thus constraining basal and oxidized low-density lipoproteins through NADPH oxidase inhibition in endothelial cells [73], eventually leading to a decrease in adipose tissue mass through activation of adiponectin receptors present mainly in lateral hypothalamic nuclei [74]. Therefore, PPAR- $\gamma$ 
agonists used in our study probably influenced the gene expression responsible for lipid and carbohydrate metabolism without affecting glycemic levels in diabetic SHRs. In parallel to our study findings, a previous study proved that pioglitazone attenuated dyslipidemia in cyclosporineinduced hypertensive rats [27], whereas in another study, Hussein et al. proved that a much greater beneficial effect of a combination of rosiglitazone and telmisartan offered more improvement in serum TGs and adiponectin [75]. Interestingly, treating diabetic rats with exogenous adiponectin and pioglitazone as full PPAR- $\gamma$ agonist produced significant attenuation of metabolic dysfunctions, as evidenced by the significant decrease in TC, TGs, and LDL, but an increase in HDL and adiponectin plasma concentrations as a similar conclusion was drawn for plasma adiponectin concentration.

4.2. Pulse Wave Velocity and Antioxidant Changes. Oxidative stress defines an imbalance between production of free radicals, its reactive metabolites, and so-called oxidants or reactive oxygen species (ROS), whereas their elimination is by protective mechanisms, referred to as antioxidants. This imbalance leads to damage of important biomolecules and cells, with potential impact on the whole organism [76]; however, oxidative stress and reactive oxygen derivatives further aggravate diabetes and hypertension [7]. In SHR, oxidative stress appears to be the cause of hypertension development on a larger scale, and the major effect of PPAR- $\gamma$ activation is the reduction of oxidative stress levels [77]. Recent epidemiological studies together with human diabetic models have suggested an association between adiponectin concentration and oxidative stress; thus, decreased circulating adiponectin levels predominates in increased oxidative stress, which is closely linked with diabetic complications $[1,2,3,78]$ and a key feature of increased production of ROS and proinflammatory pathways [11]. Reactive oxygen species, including reactive nitrogen species, hydrogen peroxide, superoxide, and hydroxyl anions, are the most significant $\mathrm{O}_{2}$ derivate, which impacts vascular biology. Moreover, vascular fibrosis also determines the vascular structural modifications in extracellular matrix (ECM) components, collagen types I and III, elastin, and fibronectin [79]. Production of ROS reduces bioavailability of NO due to uncoupling of eNOS, with enhanced levels of superoxide anions leading to formation of peroxynitrite, thus aggravating the impairment of eNOS activity and reducing NO production [80]. In our experiment, an imbalance between antioxidants and oxidative stress was observed in diabetic SHRs, which can be confirmed from the increased plasma levels of free radical mediated products of lipid peroxidation (MDA), decreased plasma concentration of enzymatic antioxidant SOD, and nonenzymatic antioxidant GSH. A decrease in TAC further confirms this imbalance indicating free radical production with a weak antioxidant defence system in diabetic SHRs, signifying the importance of OS as a common denominator in all these pathways.

Arterial stiffness is linked with endothelial dysfunction, whereas the pulse wave velocity (PWV) is considered a surrogate marker (a well-established index for arterial stiffness)
[25] and vascular diseases. The stiffer artery would lead to an increase in the PWV due to the persistent hyperglycemia leading to depletion of the antioxidant defence mechanism, generating free radicals [81] resulting in endothelial dysfunction and reduced vascular elasticity. Therefore, pulse wave velocity of diabetic SHRs was significantly higher as compared to control rats indicating the marked decrease in the extensibility of blood vessels in diabetic conditions leading to increased arterial stiffness.

However, we observed that exogenously administered adiponectin attenuated the arterial stiffness (PWV) of diabetic SHRs along with a decrease in SBP and MAP, which could be at least partially mediated through its potent antioxidant characteristics and was attenuated by blocking endothelial-derived nitric oxide synthase activity, suggesting that relaxant effect was possibly mediated by nitric oxide. However, the combination with pioglitazone resulted in a significantly greater decrease in PWV as compared with combined treatment of adiponectin with irbesartan and separate treatments. Previous clinical studies have demonstrated that partial PPAR- $\gamma$ agonist (ARBs) protects vascular endothelium via an increase of endothelial NO synthesis [82] and plasma adiponectin concentration [57] thus preventing endothelial dysfunction more effectively as compared to non-PPAR- $\gamma$ agonists ARBs [83]. Likewise, full PPAR- $\gamma$ agonist, pioglitazone, stimulates the production of $\mathrm{NO}$ and moderates oxidative stress through activation of signaling cascades, such as cAMP-PKA and AMPK-eNOS component [70], and by increasing glutathione levels, thus supporting the fact that AMPK serves as a major downstream molecule for adiponectin production [65].

The data from this study will add to the understanding of the combined treatment of adiponectin with full but not partial PPAR- $\gamma$ agonist in a combined model of hypertension and diabetes. The PPAR- $\gamma$ ligands have other important effects that inhibit atherosclerosis, including (1) improvement of endothelial function, (2) attenuation of vascular cell growth and migration, (3) inhibition of major transcription pathways mediating vascular inflammation, and (4) increase of reverse cholesterol transport. The specific agonists of PPAR- $\gamma$, TZDs, have demonstrated protective effects on a variety of atherosclerosis biomarkers and on surrogate measures of CVD, in addition to improved conventional measures of CVD risk [84]. Full PPAR- $\gamma$ agonist such as pioglitazone involves an adiponectin-dependent pathway, which increases adiponectin levels, ameliorating insulin resistance, increasing AMPK activation, and decreasing gluconeogenesis in the liver [19]. In contrary, angiotensin II receptor blockers may increase adiponectin production directly by activating the nuclear receptor PPAR- $\gamma$ [24] as partial PPAR agonists in vitro and in vivo [23]. RAAS blockers increase plasma adiponectin levels better as compared to other antihypertensive agents [85]. It is, therefore, likely that partial agonists such as angiotensin II receptor blockers irbesartan act in a similar way. However, full PPAR $-\gamma$ agonists (pioglitazone) not only act at the transcriptional level but also show to activate critical chaperones in the secretory pathway and to increase the release of the HMW form of adiponectin [86]. The use of blood 
pressure-lowering and antidiabetic agents in this study was capable of enhancing antioxidant potential through NOdependent or NO-independent mechanisms in combination therapy of adiponectin with pioglitazone which was significantly higher as compared to separate and irbesartan combination therapy, which could be due to the difference in partial and full agonistic activity for PPAR- $\gamma$ receptors, conferred by irbesartan and pioglitazone, respectively, and were therefore found to have greater beneficial/synergistic effects on the genetic model of hypertension with STZ-induced diabetes.

In this study, combined treatment of exogenous adiponectin with full PPAR- $\gamma$ agonist (pioglitazone) significantly attenuates the oxidative status to a larger extent as compared to cotreatment of adiponectin with irbesartan in experimentally induced diabetic SHRs. There was a marked increase in NO, SOD, and TOC plasma levels that indicates improvement in arterial stiffness with decreased oxidative stress in diabetic SHRs. Similarly, the reduced lipid peroxidation (MDA) values denote a decrease in free radical production, thus substantiating our findings and supporting our hypothesis tested. Increased antioxidant levels (SOD and GSH) imply better defence against ROS. These antioxidants protect the cells from oxidative damage, thereby decreasing the oxidative stress-mediated vascular complications through antioxidant-mediated pathways.

\section{Conclusion}

In a nut shell, exogenous adiponectin administration attenuated the vascular abnormalities, fluctuating from endothelial dysfunction to ROS production, through nitric oxide and antioxidant enzymatic properties with abrogation of arterial stiffness. Nonetheless, owing to the full PPAR- $\gamma$ agonist activity of pioglitazone, cotreatment with adiponectin significantly augmented to a larger extent with improvement in oxidative status and serum triglycerides and restoration of atrial stiffness (in vivo biomarker) with antioxidant enzymatic potential indicating a degree of synergism existing between adiponectin and pioglitazone.

\section{Data Availability}

The analyzed data have been incorporated in the tables and figures of the manuscript, whereas the values for these analyses of the data have been provided in the supplementary files submitted with the manuscript.

\section{Disclosure}

The preprint of this manuscript is available on Research Square and can be assessed at https://www.researchsquare .com/article/rs-45024/v1.

\section{Conflicts of Interest}

All authors have no competing or conflicts of interest for this study.

\section{Authors' Contributions}

All authors participated in the design, interpretation of the studies and analysis of the data, and review of the manuscript. Sheryar Afzal conceptualized the study, performed experiments, collected and analyzed experimental data, and drafted the manuscript. Edward J. Johns, Olorunfemi A. Eseyin, and Ali Attiq contributed to study design, interpretation, and data analysis and manuscript preparation.

\section{Acknowledgments}

All authors dedicate this scientific study as a tribute to late Professor Dr. Munavvar Zubaid Abdul Sattar (1955-2021), whose devotion to understanding the role and function of cardiovascular and renal hypertension and renal failure disease models is unsurpassed. He was a stalwart in the pharmacy profession, and his contributions had far and widereaching impact through his position as a respected scientis$\mathrm{t} /$ researcher. As a coauthor, he supervised the present study, provided lab facilities, and verified data analysis. The authors fully acknowledge the Universiti Sains Malaysia, Research grant \# 1001/PFARMASI/815078, and USM Fellowship from the Institute of Postgraduate Studies, Universiti Sains Malaysia (USM), Penang, Malaysia, for this work.

\section{Supplementary Materials}

Supplementary files contain the individual values on days of observation for each group and parameters observed in the study and were used statistically for data expression in the manuscript. (Supplementary Materials)

\section{References}

[1] B. Stratmann and D. Tschoepe, "Heart in diabetes: not only a macrovascular disease," Diabetes Care, vol. 34, Supplement 2, pp. S138-S144, 2011.

[2] J. Li and X. Shen, "Oxidative stress and adipokine levels were significantly correlated in diabetic patients with hyperglycemic crises," Diabetology \& Metabolic Syndrome, vol. 11, no. 1, pp. 1-8, 2019.

[3] D. A. McGrowder, L. Anderson-Jackson, and T. V. Crawford, "Biochemical evaluation of oxidative stress in type 1 diabetes," Diabetes Type, vol. 1, pp. 223-248, 2013.

[4] G. Siasos, D. Tousoulis, C. Vlachopoulos et al., "The impact of oral L-arginine supplementation on acute smoking-induced endothelial injury and arterial performance," American Journal of Hypertension, vol. 22, no. 6, pp. 586-592, 2009.

[5] H. A. Hadi and J. A. Suwaidi, "Endothelial dysfunction in diabetes mellitus," Vascular Health and Risk Management, vol. 3, no. 6, pp. 853-876, 2007.

[6] G. Giacchetti, L. A. Sechi, S. Rilli, and R. M. Carey, "The reninangiotensin-aldosterone system, glucose metabolism and diabetes," Trends in Endocrinology \& Metabolism, vol. 16, no. 3, pp. 120-126, 2005

[7] M. C. Houston, "Nutraceuticals, vitamins, antioxidants, and minerals in the prevention and treatment of hypertension," Progress in Cardiovascular Diseases, vol. 47, no. 6, pp. 396449, 2005. 
[8] R. Miri, H. Saadati, P. Ardi, and O. Firuzi, "Alterations in oxidative stress biomarkers associated with mild hyperlipidemia and smoking," Food and Chemical Toxicology, vol. 50, no. 34, pp. 920-926, 2012.

[9] J. H. Kim, H. W. Baik, Y. S. Yoon et al., "Measurement of antioxidant capacity using the biological antioxidant potential test and its role as a predictive marker of metabolic syndrome," The Korean Journal of Internal Medicine, vol. 29, no. 1, pp. 31-39, 2014.

[10] A. A. R. Jallod and F. S. Kata, Estimation of adiponectin hormone and antioxidants levels in type 2 diabetic patients in Dhiqar Province, Journal of Global Pharma Technology, 2009.

[11] J. R. Hahm, M. H. Jo, R. Ullah, M. W. Kim, and M. O. Kim, "Metabolic stress alters antioxidant systems, suppresses the adiponectin receptor 1 and induces Alzheimer's like pathology in mice brain," Cell, vol. 9, no. 1, p. 249, 2020.

[12] G. Siasos, D. Tousoulis, C. Kollia et al., "Adiponectin and cardiovascular disease: mechanisms and new therapeutic approaches," Current Medicinal Chemistry, vol. 19, no. 8, pp. 1193-1209, 2012.

[13] S. Nakanishi, K. Yamane, N. Kamei, H. Nojima, M. Okubo, and N. Kohno, "A protective effect of adiponectin against oxidative stress in Japanese Americans: the association between adiponectin or leptin and urinary isoprostane," Metabolism, vol. 54, no. 2, pp. 194-199, 2005.

[14] T. Yamauchi, Y. Nio, T. Maki et al., "Targeted disruption of AdipoR1 and AdipoR2 causes abrogation of adiponectin binding and metabolic actions," Nature Medicine, vol. 13, no. 3, pp. 332-339, 2007.

[15] P. Zhang, Y. Wang, Y. Fan, Z. Tang, and N. Wang, "Overexpression of adiponectin receptors potentiates the antiinflammatory action of subeffective dose of globular adiponectin in vascular endothelial cells," Arteriosclerosis, Thrombosis, and Vascular Biology, vol. 29, no. 1, pp. 67-74, 2009.

[16] S. Takatori, Y. Zamami, N. Hashikawa-Hobara, and H. Kawasaki, "Insulin resistance-induced hypertension and a role of perivascular CGRPergic nerves," Current Protein and Peptide Science, vol. 14, no. 4, pp. 275-281, 2013.

[17] K. C. Choi, O. H. Ryu, K. W. Lee et al., "Effect of PPAR- $\alpha$ and $-\gamma$ agonist on the expression of visfatin, adiponectin, and TNF$\alpha$ in visceral fat of OLETF rats," Biochemical and Biophysical Research Communications, vol. 336, no. 3, pp. 747-753, 2005.

[18] H. Yki-Järvinen, “Thiazolidinediones,” New England Journal of Medicine, vol. 351, no. 11, pp. 1106-1118, 2004.

[19] T. Kadowaki, T. Yamauchi, N. Kubota, K. Hara, K. Ueki, and $\mathrm{K}$. Tobe, "Adiponectin and adiponectin receptors in insulin resistance, diabetes, and the metabolic syndrome," The Journal of Clinical Investigation, vol. 116, no. 7, pp. 1784-1792, 2006.

[20] S. Afzal, M. A. Sattar, S. Akhtar et al., "Effect of pioglitazone on vasopressor responses to adrenergic agonists and angiotensin II in diabetic and non-diabetic spontaneously hypertensive rats," Pakistan Journal of Pharmaceutical Sciences, vol. 31, no. 3, pp. 747-754, 2018.

[21] N. Kubota, Y. Terauchi, T. Kubota et al., "Pioglitazone Ameliorates Insulin Resistance and Diabetes by Both Adiponectindependent and -independent Pathways*," Journal of Biological Chemistry, vol. 281, no. 13, pp. 8748-8755, 2006.

[22] Y. Baykal, M. I. Yilmaz, T. Celik et al., "Effects of antihypertensive agents, alpha receptor blockers, beta blockers, angiotensin-converting enzyme inhibitors, angiotensin receptor blockers and calcium channel blockers, on oxidative stress," Journal of Hypertension, vol. 21, no. 6, pp. 12071211, 2003.

[23] S. C. Benson, H. A. Pershadsingh, C. I. Ho et al., "Identification of telmisartan as a unique angiotensin II receptor antagonist with selective PPAR $\gamma$-modulating activity," Hypertension, vol. 43, no. 5, pp. 993-1002, 2004.

[24] R. Clasen, M. Schupp, A. Foryst-Ludwig et al., "PPAR $\gamma$-activating angiotensin type-1 receptor blockers induce adiponectin," Hypertension, vol. 46, no. 1, pp. 137-143, 2005.

[25] M. Willemet, P. Chowienczyk, and J. Alastruey, "A database of virtual healthy subjects to assess the accuracy of foot-to-foot pulse wave velocities for estimation of aortic stiffness," American Journal of Physiology-Heart and Circulatory Physiology, vol. 309, no. 4, pp. H663-H675, 2015.

[26] C. Vlachopoulos, F. Kosmopoulou, N. Alexopoulos, N. Ioakeimidis, G. Siasos, and C. Stefanadis, "Acute mental stress has a prolonged unfavorable effect on arterial stiffness and wave reflections," Psychosomatic Medicine, vol. 68, no. 2, pp. 231-237, 2006.

[27] M. M. El-Mas, H. M. El-Gowelli, K. S. Abd-Elrahman, E. I. Saad, A.-G. A. Abdel-Galil, and A. A. Abdel-Rahman, "Pioglitazone abrogates cyclosporine-evoked hypertension via rectifying abnormalities in vascular endothelial function," Biochemical Pharmacology, vol. 81, no. 4, pp. 526-533, 2011.

[28] B. Ramesh and K. Pugalendi, "Antihyperglycemic effect of umbelliferone in streptozotocin-diabetic rats," Journal of Medicinal Food, vol. 9, no. 4, pp. 562-566, 2006.

[29] X. Jiang, D. Song, B. Ye et al., "Effect of intermittent administration of adiponectin on bone regeneration following mandibular osteodistraction in rabbits," Journal of Orthopaedic Research, vol. 29, no. 7, pp. 1081-1085, 2011.

[30] J. R. Requena, M. X. Fu, M. U. Ahmed, A. J. Jenkins, T. J. Lyons, and S. R. Thorpe, "Lipoxidation products as biomarkers of oxidative damage to proteins during lipid peroxidation reactions," Nephrology Dialysis Transplantation, vol. 11, supp5, pp. 48-53, 1996.

[31] Y. Oyanagui, "Reevaluation of assay methods and establishment of kit for superoxide dismutase activity," Analytical Biochemistry, vol. 142, no. 2, pp. 290-296, 1984.

[32] T. Finkel and N. J. Holbrook, "Oxidants, oxidative stress and the biology of ageing," Nature, vol. 408, no. 6809, pp. 239247, 2000.

[33] Z. Duračková and A. Gvozdjáková, "Oxidants, antioxidants and oxidative stress," Mitochondrial Medicine, vol. 1, pp. 1954, 2008.

[34] J. E. Hall, Guyton and Hall Textbook of Medical Physiology eBook, Elsevier Health Sciences, 2010.

[35] K. R. L. Anand Swarup, M. A. Sattar, N. A. Abdullah et al., "Effect of dragon fruit extract on oxidative stress and aortic stiffness in streptozotocin-induced diabetes in rats," Pharmacognosy Research, vol. 2, no. 1, pp. 31-35, 2010.

[36] T. S. Frode and Y. S. Medeiros, "Animal models to test drugs with potential antidiabetic activity," Journal of Ethnopharmacology, vol. 115, no. 2, pp. 173-183, 2008.

[37] M. Mythili, R. Vyas, G. Akila, and S. Gunasekaran, "Effect of streptozotocin on the ultrastructure of rat pancreatic islets," Microscopy Research and Technique, vol. 63, no. 5, pp. 274$281,2004$.

[38] R. M. Touyz, "Reactive oxygen species, vascular oxidative stress, and redox signaling in hypertension: what is the 
clinical significance?," Hypertension, vol. 44, no. 3, pp. 248252, 2004.

[39] N. Vrbjar, S. Strelková, M. Štefek, Z. Kyselová, and A. Gajdošíková, "Effect of the pyridoindole antioxidant stobadine on sodium handling of renal $\mathrm{Na}, \mathrm{K}$-ATPase in rats with streptozotocin-induced diabetes," Acta Diabetologica, vol. 41, no. 4, pp. 172-178, 2004.

[40] M. A. Khan, M. A. Sattar, N. A. Abdullah et al., "Functional subtypes of renal \&alpha; $<$ sub $>1</$ sub $>$-Adrenoceptor in spontaneously hypertensive rats with streptozotocin-induced experimental diabetic nephropathy," Kidney and Blood Pressure Research, vol. 32, no. 5, pp. 349-359, 2009.

[41] X.-x. Yin, Y.-d. Zhang, J.-p. Shen et al., "Protective effects of bendazac lysine on early experimental diabetic nephropathy in rats," Acta Pharmacologica Sinica, vol. 26, no. 6, pp. 721728, 2005.

[42] V. Gross, U. Gnüchtel, and C. Kreher, "Erythrocyte sodium metabolism and renal sodium excretion in spontaneously hypertensive rats (SHR) and related normotensive rats (WKY)," Physiologia Bohemoslovaca, vol. 36, no. 5, pp. 477480, 1987.

[43] P. Lijnen and V. Petrov, "Induction of cardiac fibrosis by aldosterone," Journal of Molecular and Cellular Cardiology, vol. 32, no. 6, pp. 865-879, 2000.

[44] M. E. Cooper, F. Bonnet, M. Oldfield, and K. Jandeleit-Dahm, "Mechanisms of diabetic vasculopathy: an overview," American Journal of Hypertension, vol. 14, no. 5, pp. 475-486, 2001.

[45] W. T. Wong, S. L. Wong, X. Y. Tian, and Y. Huang, "Endothelial dysfunction: the common consequence in diabetes and hypertension," Journal of Cardiovascular Pharmacology, vol. 55, no. 4, pp. 300-307, 2010.

[46] P. Sharma, S. Mishra, P. Ajmera, and S. Mathur, "Oxidative stress in metabolic syndrome," Indian Journal of Clinical Biochemistry, vol. 20, no. 1, pp. 145-149, 2005.

[47] D. Laight, M. Carrier, and E. Änggård, “Antioxidants, diabetes and endothelial dysfunction," Cardiovascular Research, vol. 47, no. 3, pp. 457-464, 2000.

[48] H. Vlassara, H. Fuh, Z. Makita, S. Krungkrai, A. Cerami, and R. Bucala, "Exogenous advanced glycosylation end products induce complex vascular dysfunction in normal animals: a model for diabetic and aging complications," Proceedings of the National Academy of Sciences, vol. 89, no. 24, pp. 1204312047, 1992.

[49] U. Hink, N. Tsilimingas, M. Wendt, and T. Münzel, "Mechanisms underlying endothelial dysfunction in diabetes mellitus," Treatments in Endocrinology, vol. 2, no. 5, pp. 293-304, 2003.

[50] T. Quaschning, B. Hocher, S. Rühl et al., "Vasopeptidase inhibition normalizes blood pressure and restores endothelial function in renovascular hypertension," Kidney and Blood Pressure Research, vol. 29, no. 6, pp. 351-359, 2007.

[51] A. Armenia, M. A. Sattar, N. A. Abdullah, M. A. H. Khan, and E. J. Johns, "Functional subtypes of renal $\alpha_{1}$-adrenoceptor in diabetic and non-diabetic 2K1C Goldblatt renovascular hypertension," Acta Pharmacologica Sinica, vol. 29, no. 5, pp. 564572, 2008.

[52] K. Ichinose, Y. Maeshima, Y. Yamamoto et al., "Antiangiogenic endostatin peptide ameliorates renal alterations in the early stage of a type 1 diabetic nephropathy model," Diabetes, vol. 54, no. 10, pp. 2891-2903, 2005.

[53] J. Majithiya, A. Paramar, and R. Balaraman, "Pioglitazone, a PPAR? agonist, restores endothelial function in aorta of streptozotocin-induced diabetic rats," Cardiovascular Research, vol. 66, no. 1, pp. 150-161, 2005.

[54] R. M. Pathapati, M. Rajesh Kumar, B. R. Chirra et al., "Acute effects of two angiotensin receptor blockers on vascular hemodynamics, arterial stiffness, and oxidative stress in patients with mild to moderate hypertension: an open label parallel group study," ISRN Vascular Medicine, vol. 2013, 5 pages, 2013.

[55] D. Vidotti, D. Casarini, P. Cristovam, C. Leite, N. Schor, and M. Boim, "High glucose concentration stimulates intracellular renin activity and angiotensin II generation in rat mesangial cells," American Journal of Physiology-Renal Physiology, vol. 286, no. 6, pp. F1039-F1045, 2004.

[56] U. Kintscher and T. Unger, "Vascular protection in diabetes: a pharmacological view of angiotensin II type 1 receptor blockers," Acta Diabetologica, vol. 42, no. S1, pp. s26-s32, 2005.

[57] R. Clasen, M. Schupp, A. Foryst-Ludwig et al., "PPARgammaactivating angiotensin type-1 receptor blockers induce adiponectin," Hypertension, vol. 46, no. 1, pp. 137-143, 2005.

[58] G. A. Eppel, K. M. Denton, S. C. Malpas, and R. G. Evans, "Nitric oxide in responses of regional kidney perfusion to renal nerve stimulation and renal ischaemia," Pflügers Archiv, vol. 447, no. 2, pp. 205-213, 2003.

[59] S. Afzal, M. A. Sattar, E. J. Johns et al., "Interaction between irbesartan, peroxisome proliferator-activated receptor (PPAR- $\gamma$ ), and adiponectin in the regulation of blood pressure and renal function in spontaneously hypertensive rats," Journal of Physiology and Biochemistry, vol. 72, no. 4, pp. 593-604, 2016.

[60] X. Kong, M. Z. Ma, L. Qin et al., "Pioglitazone enhances the blood pressure-lowering effect of losartan via synergistic attenuation of angiotensin II-induced vasoconstriction," Journal of Renin-Angiotensin-Aldosterone System, vol. 15, no. 3, pp. 259-270, 2014.

[61] J. Ran, T. Hirano, T. Fukui et al., "Angiotensin II infusion decreases plasma adiponectin level via its type 1 receptor in rats: an implication for hypertension-related insulin resistance," Metabolism, vol. 55, no. 4, pp. 478-488, 2006.

[62] K. K. Cheng, K. S. Lam, Y. Wang et al., "Adiponectin-induced endothelial nitric oxide synthase activation and nitric oxide production are mediated by APPL1 in endothelial cells," Diabetes, vol. 56, no. 5, pp. 1387-1394, 2007.

[63] M. Liu, L. Zhou, A. Xu et al., "A disulfide-bond A oxidoreductase-like protein (DsbA-L) regulates adiponectin multimerization," Proceedings of the National Academy of Sciences, vol. 105, no. 47, pp. 18302-18307, 2008.

[64] S. Oparil, "The sympathetic nervous system in clinical and experimental hypertension," Kidney International, vol. 30, no. 3, pp. 437-452, 1986.

[65] Z. V. Wang and P. E. Scherer, "Adiponectin, cardiovascular function, and hypertension," Hypertension, vol. 51, no. 1, pp. 8-14, 2008.

[66] C. Kusminski, P. G. McTernan, T. Schraw et al., "Adiponectin complexes in human cerebrospinal fluid: distinct complex distribution from serum," Diabetologia, vol. 50, no. 3, pp. 634642, 2007.

[67] M. Tanida, J. Shen, Y. Horii et al., "Effects of adiponectin on the renal sympathetic nerve activity and blood pressure in rats," Experimental Biology and Medicine, vol. 232, no. 3, pp. 390-397, 2007.

[68] P. J. Havel, T. M. Hahn, D. K. Sindelar et al., "Effects of streptozotocin-induced diabetes and insulin treatment on the 
hypothalamic melanocortin system and muscle uncoupling protein 3 expression in rats," Diabetes, vol. 49, no. 2, pp. 244-252, 2000.

[69] P. M. Thulé, A. G. Campbell, D. J. Kleinhenz et al., "Hepatic insulin gene therapy prevents deterioration of vascular function and improves adipocytokine profile in STZ-diabetic rats," American Journal of Physiology-Endocrinology and Metabolism., vol. 290, no. 1, pp. E114-E122, 2006.

[70] W. T. Wong, X. Y. Tian, A. Xu et al., "Adiponectin is required for PPAR $\gamma$-mediated improvement of endothelial function in diabetic mice," Cell Metabolism, vol. 14, no. 1, pp. 104-115, 2011.

[71] H. Gao, T. Fall, R. M. van Dam et al., "Evidence of a causal relationship between adiponectin levels and insulin sensitivity a Mendelian randomization study," Diabetes, vol. 62, no. 4, pp. 1338-1344, 2013.

[72] W. Zhu, K. K. Cheng, P. M. Vanhoutte, K. S. Lam, and A. Xu, "Vascular effects of adiponectin: molecular mechanisms and potential therapeutic intervention," Clinical Science, vol. 114, no. 5, pp. 361-374, 2008.

[73] H. Motoshima, X. Wu, K. Mahadev, and B. J. Goldstein, “Adiponectin suppresses proliferation and superoxide generation and enhances eNOS activity in endothelial cells treated with oxidized LDL," Biochemical and Biophysical Research Communications, vol. 315, no. 2, pp. 264-271, 2004.

[74] E. Guillod-Maximin, A. F. Roy, C. M. Vacher et al., "Adiponectin receptors are expressed in hypothalamus and colocalized with proopiomelanocortin and neuropeptide $\mathrm{Y}$ in rodent arcuate neurons," Journal of Endocrinology, vol. 200, no. 1, pp. 93-105, 2009.

[75] A. E.-A. M. Hussein, N. M. Omar, H. Sakr, A. Z. Elsamanoudy, and D. Shaheen, "Modulation of metabolic and cardiac dysfunctions by insulin sensitizers and angiotensin receptor blocker in rat model of type 2 diabetes mellitus," Canadian Journal of Physiology and Pharmacology, vol. 89, no. 3, pp. 216-226, 2011.

[76] Z. Duračková, "Some current insights into oxidative stress," Physiological Research, vol. 59, no. 4, pp. 459-469, 2010.

[77] M. Kvandova, M. Barancik, P. Balis, A. Puzserova, M. Majzunova, and I. Dovinova, "The peroxisome proliferator-activated receptor gamma agonist pioglitazone improves nitric oxide availability, renin-angiotensin system and aberrant redox regulation in the kidney of prehypertensive rats," Journal of Physiology and Pharmacology, vol. 69, no. 2, pp. 10-26402, 2018.

[78] S.-J. Chen, C.-H. Yen, Y.-C. Huang, B.-J. Lee, S. Hsia, and P.T. Lin, "Relationships between inflammation, adiponectin, and oxidative stress in metabolic syndrome," PloS one, vol. 7, no. 9, article e45693, 2012.

[79] A. Virdis, E. Duranti, and S. Taddei, "Oxidative stress and vascular damage in hypertension: role of angiotensin II," International Journal of Hypertension, vol. 2011, 7 pages, 2011.

[80] I. Grattagliano, V. O. Palmieri, P. Portincasa, A. Moschetta, and G. Palasciano, "Oxidative stress-induced risk factors associated with the metabolic syndrome: a unifying hypothesis," The Journal of Nutritional Biochemistry, vol. 19, no. 8, pp. 491-504, 2008.

[81] A. S. De Vriese, T. J. Verbeuren, J. Van de Voorde, N. H. Lameire, and P. M. Vanhoutte, "Endothelial dysfunction in diabetes," British Journal of Pharmacology, vol. 130, no. 5, pp. 963-974, 2000.
[82] N. Kobayashi, T. Ohno, K. Yoshida et al., "Cardioprotective mechanism of telmisartan via PPAR-eNOS pathway in dahl salt-sensitive hypertensive rats," American Journal of Hypertension, vol. 21, no. 5, pp. 576-581, 2008.

[83] S. Takai, D. Jin, M. Kimura et al., "Inhibition of Vascular Angiotensin-Converting Enzyme by Telmisartan via the Peroxisome Proliferator -Activated Receptor $\gamma$ Agonistic Property in Rats," Hypertension Research, vol. 30, no. 12, pp. 1231-1237, 2007.

[84] P. Sarafidis and A. Lasaridis, "Actions of peroxisome proliferator-activated receptors $-\gamma$ agonists explaining a possible blood pressure-lowering effect," American Journal of Hypertension, vol. 19, no. 6, pp. 646-653, 2006.

[85] M. I. Yilmaz, A. Sonmez, K. Caglar et al., "Effect of antihypertensive agents on plasma adiponectin levels in hypertensive patients with metabolic syndrome," Nephrology, vol. 12, no. 2, pp. 147-153, 2007.

[86] Z. V. Wang, T. D. Schraw, J.-Y. Kim et al., "Secretion of the adipocyte-specific secretory protein adiponectin critically depends on thiol-mediated protein retention," Molecular and Cellular Biology, vol. 27, no. 10, pp. 3716-3731, 2007. 